\title{
Mode competition of rotating waves in reflection-symmetric Taylor-Couette flow
}

\author{
By J. ABSHAGEN ${ }^{1}$, J. M. LOPEZ ${ }^{2}$, F. MARQUES \\ AND G. PFISTER ${ }^{1}$ \\ ${ }^{1}$ Institute of Experimental and Applied Physics, University of Kiel, 24105 Kiel, Germany \\ ${ }^{2}$ Department of Mathematics and Statistics, Arizona State University, Tempe, AZ 85287, USA \\ ${ }^{3}$ Departament de Física Aplicada, Universitat Politècnica de Catalunya, \\ 08034, Barcelona, Spain
}

(Received 3 November 2004 and in revised form 3 May 2005)

We report on the results of a combined experimental and numerical study on mode interactions of rotating waves in Taylor-Couette flow. Our work shows that rotating waves which originate at a Hopf bifurcation from the steady axisymmetric Taylor vortex flow interact with this axisymmetric flow in a codimension-two fold-Hopf bifurcation. This interaction gives rise to an (unstable) low-frequency modulated wave via a subcritical Neimark-Sacker bifurcation from the rotating wave. At higher Reynolds numbers, a complicated mode interation between stable modulated waves originating at a different Neimark-Sacker bifurcation and a pair of symmetrically related rotating waves that originate at a cyclic pitchfork bifurcation is found to organize complex $Z_{2}$-symmetry breaking of rotating waves via global bifurcations. In addition to symmetry breaking of rotating waves via a (local) cyclic pitchfork bifurcation, we found symmetry breaking of modulated waves via a saddle-nodeinfinite-period (SNIP) global bifurcation. Tracing these local and global bifurcation curves in Reynolds number/aspect ratio parameter space toward their apparant merging point, unexpected complexity arises in the bifurcation structure involving non-symmetric two-tori undergoing saddle-loop homoclinic bifurcations. The close agreement between the numerics and the experiment is indicative of the robustness of the observed complex dynamics.

\section{Introduction}

Bifurcation theory has long been a very useful tool in the analysis of complex nonlinear hydrodynamics. Examples arise from convection, such as Rayleigh-Bénard convection (Tuckerman \& Barkley 1988; Kevrekidis et al. 1994) and electroconvection in liquid crystals (Peacock \& Mullin 2001), and from rotating fluids, such as TaylorCouette flow (Mullin 1991, 1993; Egbers \& Pfister 2000; Lopez, Marques \& Fernando 2004a) and lid-driven cavities (Blackburn \& Lopez 2002; Marques, Lopez \& Shen 2002; Lopez et al. 2002a; Nore et al. 2003; Lopez \& Marques 2004). Symmetries determine the solution set close to a bifurcation and symmetry-breaking bifurcations have been found to be an important mechanism for the appearance of multiple solutions in fluid flows (Benjamin \& Mullin 1982; Crawford \& Knobloch 1991; Chossat \& Iooss 1994). In symmetric systems, any solution to which a symmetry operator is applied is also a solution, and if that solution is unchanged then the solution is symmetric. Otherwise, the solutions are distinct and symmetrically 
related. In such systems, bifurcations can be interpreted as either symmetry breaking (restoring) or symmetry preserving.

Complex hydrodynamics is often organized by global bifurcations which originate from the local bifurcation structure. In particular, homoclinic and heteroclinic bifurcations have been found to play a crucial role in hydrodynamics. Mode interaction provides a generic mechanism to organize homoclinic and heteroclinic bifurcations in the vicinity of a multiple local bifurcation point. Moreover, lowfrequency dynamics often arise from a Neimark-Sacker bifurcation (Hopf-like bifurcation from a limit cycle) as a result of mode interaction. Therefore, in-depth knowledge of mode interaction is important for the understanding of the organization of complex dynamics in fluid flows.

Taylor-Couette flow between two concentric rotating cylinders is one of the classical hydrodynamic systems for the study of bifurcation events and the transition to turbulence. The 'standard' set-up consists of a rotating inner cylinder and stationary outer cylinder and endwalls. The symmetry group of this system is $S O(2) \times Z_{2}$, corresponding to invariance to rotations about the axis, $S O(2)$, and reflection about the axial mid-plane, $Z_{2}$. There are three non-dimensional governing parameters: the Reynolds number $R e$, the radius ratio $\eta$, and the length-to-gap aspect ratio $\Gamma$.

From the pioneering work of Benjamin and Mullin (Benjamin 1978a, $b$; Mullin 1982) it has become evident that endwalls are dominant for the bifurcation behaviour of the Taylor-Couette system. In fact, the axial translation invariance that is inherent in models assuming periodic boundary conditions is completely destroyed by the presence of endwalls; the transition to axisymmetric Taylor cells is smooth with increasing Reynolds number, rather than the pitchfork of revolution bifurcation found in the idealized periodic model (Crawford \& Knobloch 1991). With physical endwall boundary conditions, the primary bifurcations to axisymmetric Taylor vortex flows are saddle-node bifurcations that are organized by codimension-two cusp bifurcations resulting from mode interchange processes between flow states with $2 \mathrm{~N}$ and $2 \mathrm{~N}+2$ cells, with $2 N$ being of order $\Gamma$.

Subsequent numerical and experimental work revealed that crucial mechanisms for the organization of the axisymmetric flow are $Z_{2}$ symmetry-breaking pitchfork bifurcations (Mullin, Toya \& Tavener 2002). Mode interaction of symmetry-breaking bifurcations and saddle-node (fold) bifurcations originating from mode interchange processes give rise to low-frequency dynamics resulting from a Hopf bifurcation (Mullin, Tavener \& Cliffe 1989) and the appearance of complex dynamics due to Shil'nikov mechanisms (Price \& Mullin 1991; Mullin 1991). The loss of axisymmetry, i.e. $S O(2)$ symmetry breaking, occurs primarly via Hopf bifurcations which lead to rotating waves with azimuthal wavenumbers $m$ (Knobloch 1994), and they may be either reflection symmetric or have a spatio-temporal reflection symmetry composed of a reflection together with an appropriate rotation (DiPrima \& Swinney 1981; Tagg 1994). Rotating waves are periodic solutions which are special in the sense that the time-dependence is a drift in the direction of the $S O(2)$ symmetry, i.e. a precession, and are better described as relative equilibria. Near a relative equilibrium the drift dynamics associated with the precession is trivial and decouples from the dynamics orthogonal to the relative equilibrium. As a result the bifurcations from relative equilibria can be analysed in two steps, describing first the bifurcations associated with the orthogonal dynamics, and then adding the corresponding drift along the rotating wave (Krupa 1990). Subsequent instabilities of rotating waves to quasiperiodic flows have been explored experimentally and found to lead to a multitude of very-low-frequency states via a variety of global bifurcations, the details depending on 


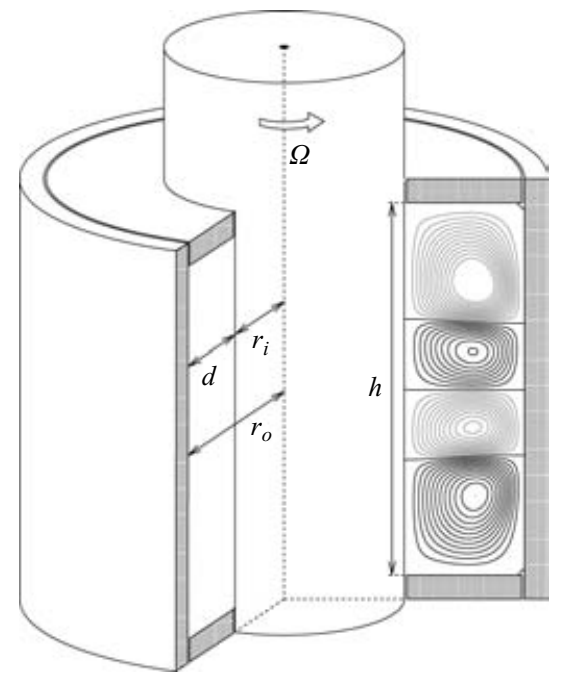

FIGURE 1. Schematic of the flow apparatus.

all three governing parameters (Gerdts et al. 1994; von Stamm et al. 1996; Abshagen, Pfister \& Mullin 2001; Abshagen et al. 2005).

In this study, we investigate both numerically and experimentally the mode competition of rotating waves. The aim is to clarify the origin of low-frequency dynamics and the role global bifurcations play in Taylor-Couette flow. In order to reduce the number of possible multiple solutions and their interactions, the aspect ratio $\Gamma$ has to be choosen to be small, between about 3 and 4 , so that the mode competition is between basic states with two and four cells. A fixed wide-gap radius ratio of $\eta=0.5$ is used throughout this study. In the wide-gap regime the sequences of bifurcations are more spaced out in $(\Gamma, R e)$ parameter space than they are in the narrow-gap $\eta \rightarrow 1$ regime.

\section{Navier-Stokes equations and the numerical scheme}

We consider an incompressible flow confined in an annulus of inner radius $r_{i}$ and outer radius $r_{o}$ and length $h$, driven by the constant rotation of the inner cylinder at $\Omega \mathrm{rad} \mathrm{s}^{-1}$. The system is non-dimensionalized using the gap, $d=r_{o}-r_{i}$, as the length scale and the diffusive time across the gap, $d^{2} / v$, as the time scale (where $v$ is the fluid's kinematic viscosity). The equations governing the flow are the Navier-Stokes equations together with initial and boundary conditions. In cylindrical coordinates, $(r, \theta, z)$, we denote the non-dimensional velocity vector and pressure by $\boldsymbol{u}=(u, v, w)^{T}$ and $p$, respectively. The system is governed by three non-dimensional parameters, two geometric and one dynamic:

$$
\begin{aligned}
& \text { radius ratio: } \quad \eta=r_{i} / r_{o} \text {, } \\
& \text { annulus aspect ratio: } \quad \Gamma=h / d \text {, } \\
& \text { Reynolds number: } \quad \operatorname{Re}=\Omega d r_{i} / \nu \text {. }
\end{aligned}
$$

In this study, we fix $\eta=0.5$ and vary $R e$ and $\Gamma$, both in the numerical computations and the experiments. A schematic of the flow geometry, with an insert showing the streamlines for a steady axisymmetric solution at $R e=400, \Gamma=3.10$ is given in figure 1 . 
The governing equations are the (non-dimensional) Navier-Stokes equations

$$
\partial \boldsymbol{u} / \partial t+(\boldsymbol{u} \cdot \nabla) \boldsymbol{u}=-\nabla p+\nabla^{2} \boldsymbol{u}, \quad \nabla \cdot \boldsymbol{u}=0,
$$

subject to no-slip boundary conditions. Specifically, $u=v=w=0$ on all stationary boundaries, i.e. at the outer cylinder, $r=r_{o} / d=1 /(1-\eta)=2$, and the top and bottom endwalls $z= \pm 0.5 h / d= \pm 0.5 \Gamma$. On the rotating inner cylinder, $r=r_{i} / d=$ $\eta /(1-\eta)=1, u=w=0$ and $v=R e$.

To solve (2.1), a stiffly stable semi-implicit second-order projection scheme is used, where the linear terms are treated implicitly while the nonlinear terms are explicit (see Lopez \& Shen 1998; Lopez, Marques \& Shen 2002b, for more details, including the treatment of discontinuous boundary conditions). For the space variables, we use a Legendre-Fourier approximation. More precisely, the azimuthal direction is discretized using a Fourier expansion with $N_{\theta}+1$ modes corresponding to azimuthal wavenumbers $m=0,1,2, \ldots N_{\theta}$, while the axial and radial directions are discretized with a Legendre expansion. For example, the spectral expansion for the axial velocity component is

$$
w(r, \theta, z, t)=\sum_{i=0}^{N_{z}} \sum_{j=0}^{N_{r}} \sum_{k=-N_{\theta}}^{N_{\theta}} w_{i, j, k}(t) \phi_{i}\left(\frac{2 z}{\Gamma}\right) \psi_{j}\left(2 r-\frac{1+\eta}{1-\eta}\right) \mathrm{e}^{\mathrm{i} k \theta},
$$

where $\phi_{i}$ and $\psi_{j}$ are appropriate combinations of Legendre polynomials in order to satisfy boundary conditions.

The spectral convergence of the code in the radial and axial directions has already been extensively described in Lopez \& Shen (1998) for $m=0$; the convergence properties in these directions are not affected by $m \neq 0$. For the convergence in azimuth, we note that the mode of instability being investigated here leads to rotating waves with azimuthal wavenumber $m=1$. This numerical scheme has been used to investigate similar dynamics in Taylor-Couette flows with different boundary conditions and parameter regimes where resolution issues have been addressed (Lopez \& Marques 2003; Lopez, Marques \& Shen 2004b). The results presented here have 48 and 64-96 Legendre modes in the radial and axial directions, respectively, and up to 11 Fourier modes in $\theta$ (resolving up to azimuthal wavenumber $m=10$ ); the time step used is $\delta t \in\left[10^{-5}, 10^{-4}\right]$.

\section{Experimental technique}

The experimental set-up of the Taylor-Couette system used for this study consists of a fluid (silicon oil with kinematic viscosity $v=10.2 \mathrm{~mm}^{2} \mathrm{~s}^{-1}$, with an absolute uncertainty of $\pm 0.1 \mathrm{~mm}^{2} \mathrm{~s}^{-1}$ ) confined in the gap between two concentric cylinders. The outer cylinder and the top and bottom endwalls were held fixed. A phaselocked loop (PLL) circuit controlled the angular velocity of the inner cylinder, $\Omega$, to an accuracy of better than one part in $10^{-4}$ in the short term and $10^{-7}$ in the long-term average. The inner cylinder was machined from stainless steel with radius $r_{i}=(12.50 \pm 0.01) \mathrm{mm}$, while the outer cylinder, with $r_{o}=(25.00 \pm 0.01) \mathrm{mm}$, was made from optically polished glass. The temperature of the fluid was thermostatically controlled to $(21.00 \pm 0.01)^{\circ} \mathrm{C}$. The apparatus was located in an air-controlled cabinet and the laboratory was air conditioned. The distance between the endwalls, $h$, is adjustable within an accuracy of $0.01 \mathrm{~mm}$. Laser Doppler velocimetry (LDV) was used for measurements of the local flow velocity and laser light-sheet techniques were used for flow visualization. Further technical details of the experimental apparatus 


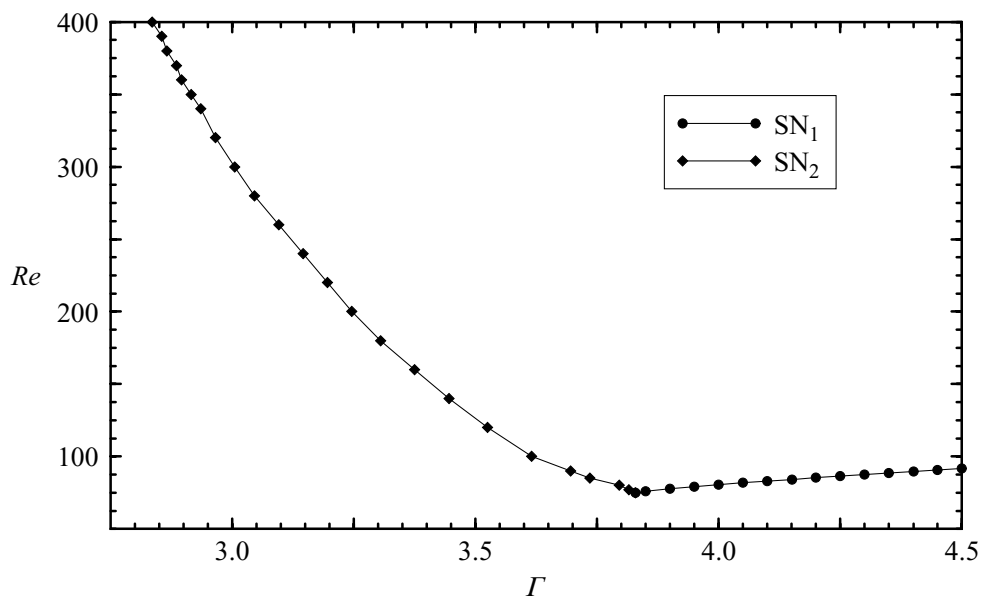

FIGURE 2. Saddle-node bifurcation curves $\mathrm{SN}_{1}$ and $\mathrm{SN}_{2}$, where $\mathrm{s}_{1}$ and $\mathrm{s}_{2}$ each undergo saddle-node bifurcations, respectively.

and measurement procedures can be found in Gerdts et al. (1994) and von Stamm et al. (1996).

\section{Basic flow states and their saddle-node bifurcations}

For $\eta=0.5$ and $\Gamma$ of order 4 , there is a broad wedged-shaped region in $(\Gamma, R e)$ parameter space, delimited by a pair of saddle-node bifurcation curves which meet at a codimension-two cusp point at $(\Gamma=3.81, R e=76)$. In this region there are three basic-state solutions (i.e. solutions which are steady and $S O(2) \times Z_{2}$ symmetric, $S O(2)$ being invariance to rotations about the axis and $Z_{2}$ invariance to reflection through the mid-plane $z=0)$; two are stable ( $\mathrm{s}_{1}$ and $\mathrm{s}_{2}$, described below) and the third $\left(\mathrm{S}_{\mathrm{M}}\right)$ is unstable. Figure 2 shows the loci of these saddle-node bifurcation curves. The two stable steady states are distinguished by the number of jets of angular momentum that issue from the boundary layer on the rotating inner cylinder; one has a single jet, denoted $\mathrm{s}_{1}$, and the other has two, denoted $\mathrm{s}_{2}$. Contour plots of the three computed components of velocity and the corresponding streamlines, as well as a flow visualization photograph of $s_{1}$ and $s_{2}$, are shown in figures 3 and 4 , respectively, both at $R e=330, \Gamma=3.0, \eta=0.5$. For $\Gamma>3.81, \mathrm{~s}_{2}$ is smoothly connected to the unique basic state as $R e \rightarrow 0$ (the Stokes flow limit), which is characterized by Ekman vortices on the endwalls, and $\mathrm{s}_{1}$ comes into existence with increasing $R e$ as the saddlenode curve $\mathrm{SN}_{1}$ is crossed. For $\Gamma<3.81, \mathrm{~s}_{1}$ is connected smoothly with the Stokes flow limit and $\mathrm{S}_{2}$ comes into existence with increasing $R e$ as the saddle-node curve $\mathrm{SN}_{2}$ is crossed.

In this study, we shall focus on the dynamics involved in the competition between bifurcated states from $\mathrm{s}_{2}$ for $\Gamma<3$.8. In this parameter regime, a rich dynamics manifests itself as $\mathrm{S}_{2}$ is followed towards the saddle-node bifurcation $\mathrm{SN}_{2}$.

\section{Hopf bifurcation to a rotating wave}

The basic state $\mathrm{s}_{2}$ loses stability via a symmetry-breaking Hopf bifurcation as $R e$ is increased beyond about 400 for $\Gamma \in(2.9,3.4)$. There are four possible types of symmetry which the resulting limit cycle may have, distinguished by the actions of the 

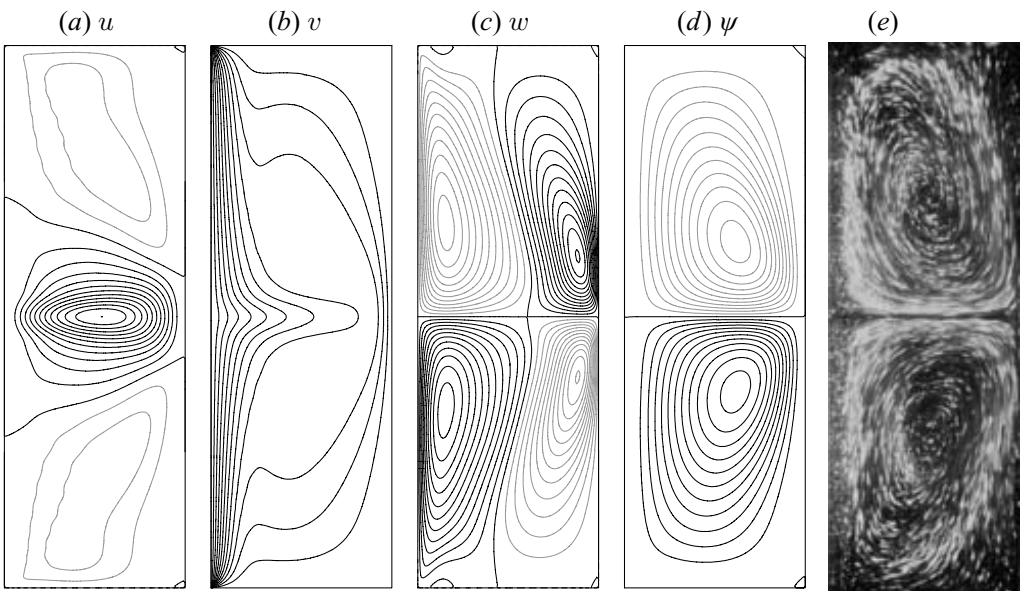

FIGURE 3. Axisymmetric steady state, $\mathrm{s}_{1}$, at $R e=330, \Gamma=3.0, \eta=0.5$; computed with $N_{z}=64$, $N_{r}=48, \delta t=10^{-4}$; there are twelve positive (black) and negative (grey) contours with the following levels: (a) $u \in[-125,125],(b) v \in[0,400],(c) w \in[-80,80]$, and $(d) \psi \in[-15,15]$; (e) corresponding visualization of the experimental flow.

(a) $u$

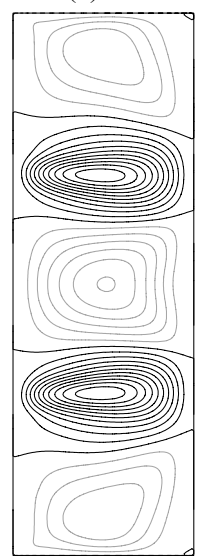

(b) $v$

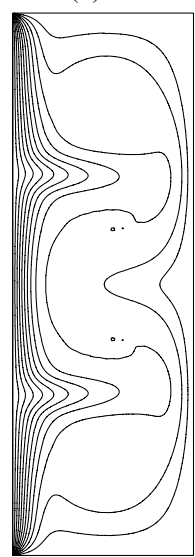

(c) $w$

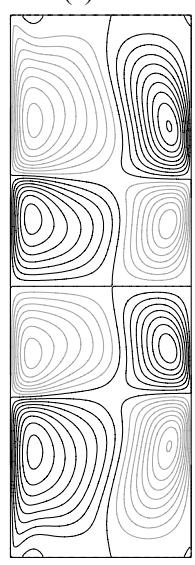

(d) $\psi$

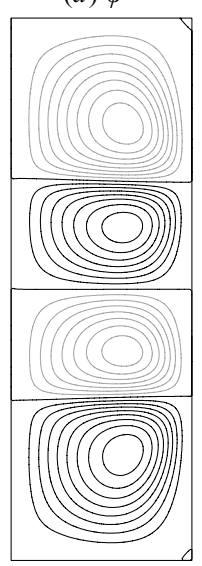

(e)

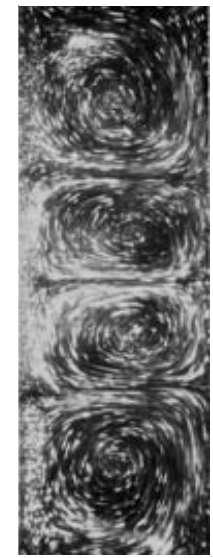

FIGURE 4. As figure 3 but for axisymmetric steady state, $s_{2}$.

two symmetry subgroups $Z_{2}$ and $S O(2)$, and characterized by $s= \pm 1$, associated with the action of $Z_{2}$, and $m \in \mathbb{Z}$ related to the action of $S O(2)$ (Lopez \& Marques 2003, 2004). If $m=0$, the action of $S O(2)$ is trivial, and the limit cycle is axisymmetric; if $s=+1$, the action of $Z_{2}$ is trivial, and the limit cycle is point-wise reflection symmetric. If $m \neq 0$, the action of $S O(2)$ is equivalent to a temporal evolution, and the limit cycle is a rotating wave with azimuthal wavenumber $m$. If $s=-1$, the action of $Z_{2}$ is equivalent to a half-period temporal evolution.

The Hopf bifurcation at which $\mathrm{S}_{2}$ loses stability, $\mathrm{H}_{2}$, leads to a limit cycle with $m=1$ and $s=-1$, a type IV limit cycle (Lopez \& Marques 2003, 2004). It is a rotating wave with precession period $\tau=2 \pi / \omega$ where $\omega$ is the Hopf frequency, and we denote it $\mathrm{RW}_{\mathrm{S}}$. $\mathrm{RW}_{\mathrm{S}}$ is invariant to a rotation about the axis through $\pi$ composed with a reflection about the mid-plane. This is an involution; applying this twice is equivalent to the action of the identity, i.e. it is a $Z_{2}$ symmetry. Figure 5 shows contours in 
(a) $\theta=0$

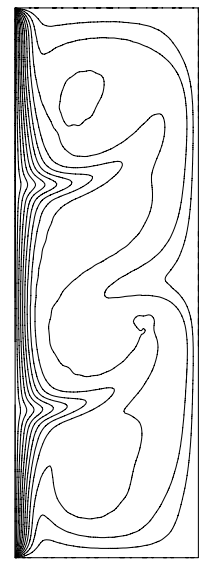

(b) $\pi / 3$

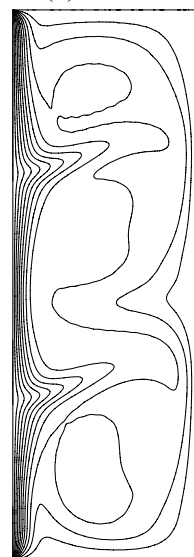

(c) $2 \pi / 3$

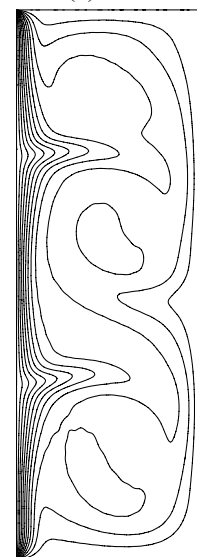

(d) $\pi$

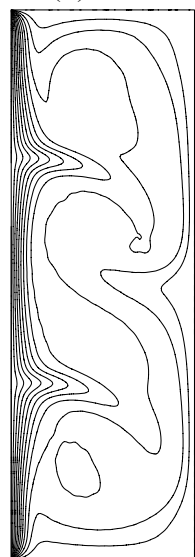

(e) $4 \pi / 3$

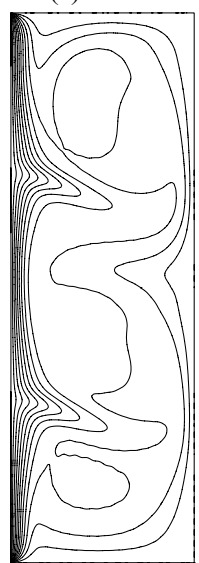

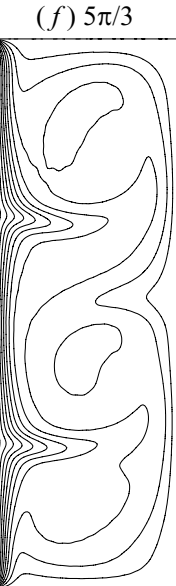

FIGURE 5. Contours of $v$ in various meridional planes, as indicated, for $\mathrm{RW}_{\mathrm{S}}$ at $R e=700$, $\Gamma=3.0, \eta=0.5$; computed with $N_{z}=96, N_{r}=48, N_{\theta}=10, \delta t=2 \times 10^{-5}$. There are twelve equispaced contours with the levels $v \in[0, R e]$.

(a) $z=-\Gamma / 3$

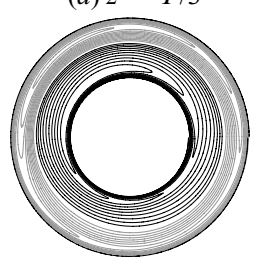

(b) $-\Gamma / 6$

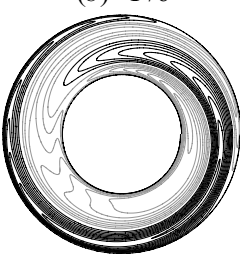

(c) 0

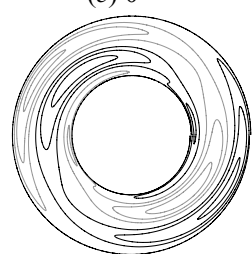

(d) $\Gamma / 6$

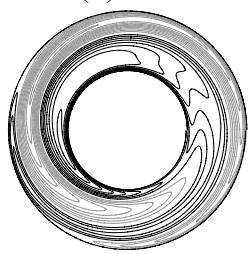

(e) $\Gamma / 3$

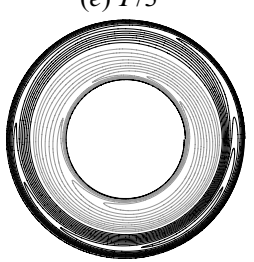

FIgure 6. Contours of $w$, at various axial levels as indicated, for $\mathrm{RW}_{\mathrm{S}}$ at $\operatorname{Re}=700, \Gamma=3.0$, $\eta=0.5$; computed with $N_{z}=96, N_{r}=48, N_{\theta}=10, \delta t=2 \times 10^{-5}$. There are twelve positive (black) and negative (gray) contours with the levels $w \in[-150,150]$.

various meridional planes around the annulus of the azimuthal velocity of a $\mathrm{RW}_{\mathrm{S}}$ solution at $R e=700$ and $\Gamma=3.0$. The in-phase tilts of the two jets issuing from the boundary on the rotating inner cylinder are clearly evident, as is the $Z_{2}$ symmetry composed of a rotation through $\pi$ together with a reflection about $z=0$. Note also that the rotation through $\pi$ (half a spatial period rotation) is equivalent a temporal evolution through half the precession period of the rotating wave.

The non-axisymmetric nature of $\mathrm{RW}_{\mathrm{S}}$ is associated with the tilting of the jets, which in the basic state $S_{2}$ issue from the boundary layer at constant $z$ (compare with figure $4 b$ ). Figure 6 shows contours of the axial velocity of the RWS solution shown in figure 5 at various axial levels $z$. Note that the jets issue from the boundary layer at about $z= \pm \Gamma / 6$, and at these $z$ levels the solution is most non-axisymmetric (the $m=1$ nature of the tilt is quite evident), and away from the jets (e.g. for $z= \pm \Gamma / 3$ ), $\mathrm{RW}_{\mathrm{S}}$ is very nearly axisymmetric. This type of $m=1$ rotating wave state is often referred to as a tilt wave.

Figure 7 shows contours of the azimuthal velocity of the RWS solution shown in figures 5 and 6 , on $(a)$ the meridional plane $\theta=0$, and $(b)$ a cylindrical surface $(\theta, z)$ at $r=r_{i} / d+0.3$, projected on a plane and compressed in azimuth by a factor of four in order to better display the structure of the tilt wave. The figure shows that both jets are in phase, that $\mathrm{RW}_{\mathrm{S}}$ is a mode $m=1$ solution, and also clearly displays the 
(a)

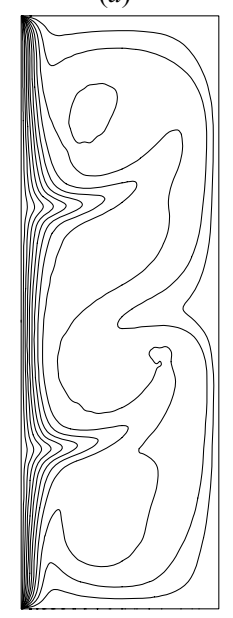

(b)

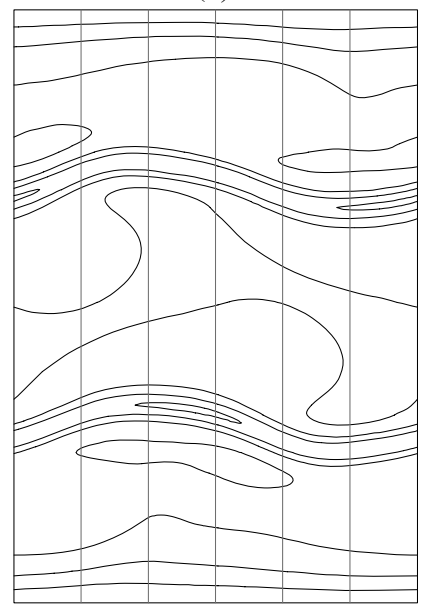

FIgURE 7. Contours of $v$ on $(a)$ the meridional plane $\theta=0$, and $(b)$ the cylindrical projection $(\theta, z)$ at $r=r_{i} / d+0.3$, for RWS at $\operatorname{Re}=700, \Gamma=3.0, \eta=0.5$. Vertical lines at angles $\theta=j \pi / 3$ for $j=1$ to 5 are included in $(b)$, which correspond to the meridional planes used in figure 5 . There are twelve equispaced contours with the levels $v \in[0, R e]$.

$Z_{2}$ symmetry of the RWS : invariance under a $z$-reflection followed by a rotation of $\pi$ around the axis: $\theta \rightarrow \theta+\pi$.

For systems with larger $\Gamma$, for which the basic state has more than two outgoing jets, their primary Hopf bifurcations (i.e. the first Hopf bifurcation from the basic state as parameters are varied) lead to rotating waves $(m \neq 0)$ with the jets tilted out-of-phase, i.e. $s=+1$ for an even number of jets and $s=-1$ for an odd number of jets. The two-jet case $s_{2}$ studied here is the only one for which the primary Hopf bifurcation leads to a rotating wave with the jets tilted in-phase, $s=-1$; the secondary Hopf bifurcation (i.e. the second Hopf bifurcation from the now unstable basic state) with $s=+1$, from the basic state $\mathrm{s}_{2}$, has not been found experimentally (or numerically). Experiments over a wide range of $\Gamma$ (up to $\Gamma=32$ ) have shown that for basic states with three or more jets, the out-of-phase mode is always the primary rotating wave, and that the critical $R e$ for the Hopf bifurcation is independent of the number of jets but depends on $\Gamma$ (von Stamm et al. 1996). For the single-jet basic state $\mathrm{s}_{1}$ at $\Gamma=0.5$, Hopf bifurcations with both $s=+1$ and $s=-1$ were found to compete as primary bifurcations when the radius ratio is larger than that considered here, $\eta \sim 0.67$ compared with the present $\eta=0.5$ (Lopez \& Marques 2003).

Figure 8 shows the saddle-node bifurcation curve $\mathrm{SN}_{2}$, together with the Hopf bifurcation curve $\mathrm{H}_{2}$ at which $\mathrm{RW}_{\mathrm{S}}$ bifurcates from $\mathrm{S}_{2}$, and a Neimark-Sacker bifurcation curve $\mathrm{NS}_{2}$ at which $\mathrm{RW}_{\mathrm{S}}$ becomes unstable. The solid (dotted) lines with the filled (open) symbols are the numerically (experimentally) determined curves.

The precession periods $\tau$ close to the onset of the Hopf bifurcation are shown in figure 9; the filled squares are the experimentally determined periods, and the open diamonds are the computationally determined values. The precession period is a small fraction of the viscous diffusion time across the annular gap, varying between about $3.5 \%$ and $2.5 \%$ of the viscous time for $\Gamma$ between about 2.9 and 3.5 . We have found that the precession period varies much less with $R e$ than it does with $\Gamma$. The agreement between the numerics and the experiments for both the critical values of the parameters and for the critical eigenvalue (inversely proportional to the 


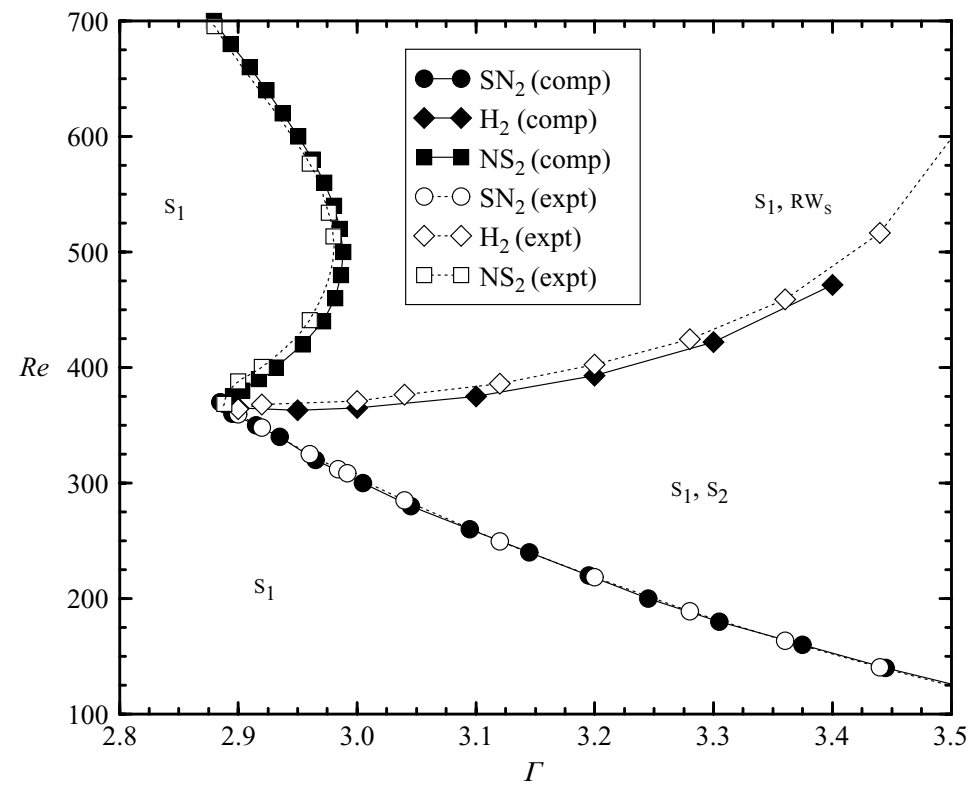

FIGURE 8. Bifurcation curves in $(\Gamma, R e)$ space, including the saddle-node $\mathrm{SN}_{2}$ (only shown up to the fold-Hopf point), the Hopf $\mathrm{H}_{2}$, and the Neimark-Sacker $\mathrm{NS}_{2}$ bifurcation curves. The stable solutions in each region are indicated. The solid (dotted) lines with the filled (open) symbols are the numerically (experimentally) determined curves.

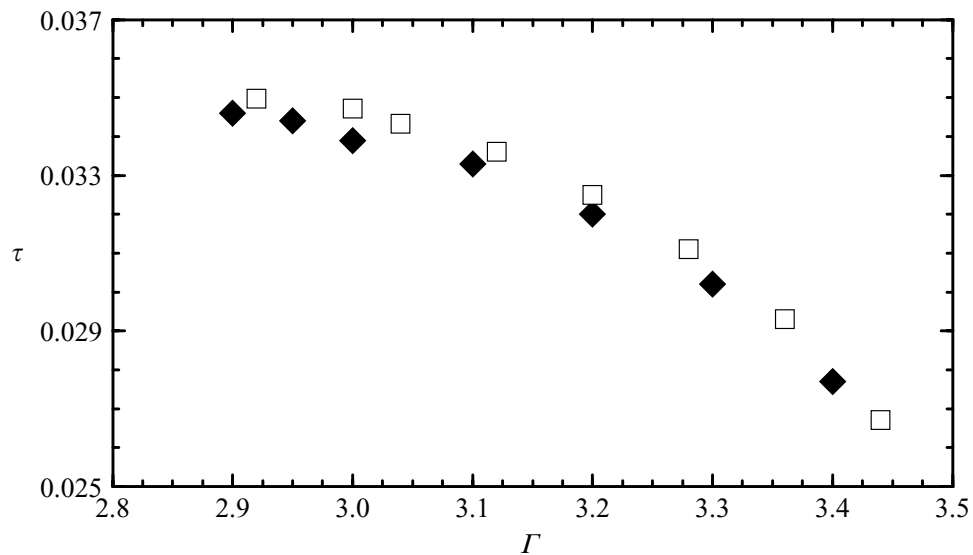

FiguRE 9. Variation with $\Gamma$ of the precession period of the rotating wave $\mathrm{RW}_{\mathrm{S}}, \tau$, very close to the Hopf bifurcation, in viscous time units $d^{2} / v$, as determined computationally (solid diamonds) and experimentally (open squares).

precession period at onset) is indicative of the precision of the experiments and the accuracy of the numerics.

The Hopf bifurcation $\mathrm{H}_{2}$ is supercritical; at onset, the amplitude squared of the rotating wave grows linearly with distance in parameter space from the bifurcation curve, and the precession period is only a weak function of the parameters. To estimate the amplitude squared, we introduce global measures of the solution which 


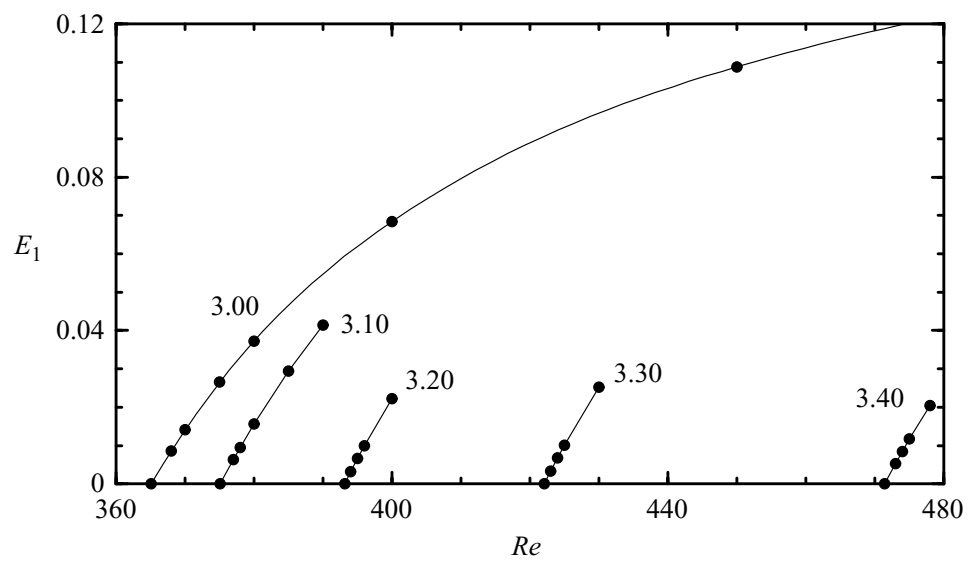

FIGURE 10. Variation of $E_{1}$ of $\mathrm{RW}_{\mathrm{S}}$ with $R e$ for $\Gamma$ as indicated.

are the (scaled) kinetic energies in the $m$ th Fourier modes of the solution:

$$
E_{m}=\frac{1}{\operatorname{Re}^{2}} \int_{z=-\Gamma / 2}^{z=\Gamma / 2} \int_{r=1}^{r=2} \boldsymbol{u}_{m} \cdot \overline{\boldsymbol{u}}_{m} r \mathrm{~d} r \mathrm{~d} z,
$$

where $\boldsymbol{u}_{m}$ is the $m$ th Fourier mode of the velocity field. For rotating waves, $E_{m}$ is constant in time. For RW,$m=1$; in figure 10 we plot $E_{1}$ as a function of $R e$ for various $\Gamma \in[3.00,3.40]$, which cover the Hopf bifurcation curve $\mathrm{H}_{2}$ shown in figure 8 . The linear growth of $E_{1}$ from zero with increasing $R e$ is clearly evident. While we do not have experimental data measuring the 'amplitude' of RWS for the present range of $\Gamma$, the supercriticality of the Hopf bifurcation leading to $\mathrm{RW}_{\mathrm{S}}$ in other parameter regimes has been determined experimentally in Gerdts et al. (1994).

The $\mathrm{SN}_{2}$ saddle-node bifurcation curve and the $\mathrm{H}_{2}$ Hopf bifurcation curve coincide at a codimension-two fold-Hopf bifurcation point at $(\Gamma=2.89, R e=360)$, from which a Neimark-Sacker $\left(\mathrm{NS}_{2}\right)$ bifurcation curve also emerges (see figure 8). The nonlinear dynamics in the neighbourhood of this fold-Hopf point are now considered.

\section{Fold-Hopf bifurcation}

In the neighbourhood of a codimension-two fold-Hopf bifurcation, the infinitedimensional phase space of the Taylor-Couette problem admits a three-dimensional centre manifold parameterized by a coordinate $x$, an amplitude $\rho$ and an angle $\phi$. The normal form is given by (Kuznetsov 1998)

$$
\left.\begin{array}{l}
\dot{x}=-\mu_{1}+x^{2}+\sigma \rho^{2}, \\
\dot{\rho}=\rho\left(-\mu_{2}+\chi x+x^{2}\right), \\
\dot{\phi}=\omega,
\end{array}\right\}
$$

where $\mu_{1}$ and $\mu_{2}$ are the normalized bifurcation parameters related to Re and $\Gamma$. The signs in (6.1) have been chosen in order to easily compare with our representation of the dynamics in $(\Gamma, R e)$-parameter space. The eigenvalues at the fold-Hopf bifurcation point $\mu_{1}=\mu_{2}=0$ are zero and $\pm \mathrm{i} \omega$. The coefficients in the normal form are $\sigma= \pm 1$, and $\chi$ and $\omega$ that depend on the parameters $\mu_{1}$ and $\mu_{2}$ and satisfy certain nondegeneracy conditions in the neighbourhood of the bifurcation: $\omega \neq 0, \chi \neq 0$. The $\phi$ equation describes a rotation around the $x$-axis with constant angular velocity, 
(6a)

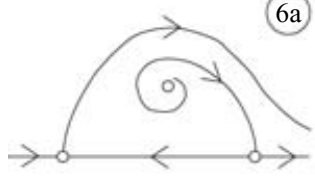

(6b)

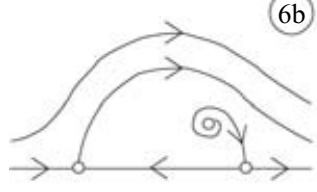

(7)

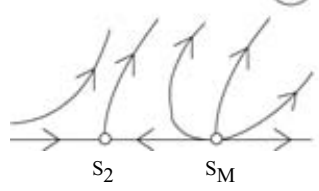

(5)

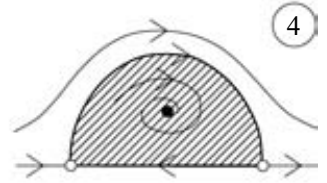

(4)

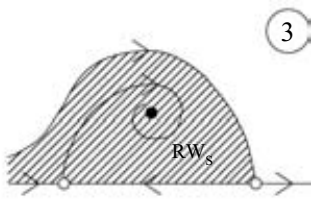

(2)

FIGURE 11. Bifurcation diagram of the fold-Hopf bifurcation, in normal form variables, corresponding to the present flow. Filled $(\bullet)$ and open $(\circ)$ dots correspond to stable and unstable solutions respectively, $\mu_{1}$ and $\mu_{2}$ are the two bifurcation parameters, $\mathrm{SN}_{2}$ is a saddle-node bifurcation curve, $\mathrm{H}_{2}$ is the Hopf bifurcation curve, $\mathrm{NS}_{2}$ is the Neimark-Sacker bifurcation curve, and 4 is the horn region of complex dynamics; the straight line inside region 4 is the heteroclinic connection predicted by the formal normal form (6.1) and shown in panel 4 as a thick line.

$\phi=\phi_{0}+\omega t$. Thus, to understand the bifurcations in (6.1), we only need to consider the planar system for $(x, \rho)$, with $\rho \geqslant 0$, which is independent of $\phi$. This system has three fixed points:

(a) $\mathrm{s}_{2}=\left(-\sqrt{\mu_{1}}, 0\right)$, which exists for $\mu_{1}>0$ and is stable iff $\mu_{2}+\chi \sqrt{\mu_{1}}<0$;

(b) $\mathrm{S}_{\mathrm{M}}=\left(\sqrt{\mu_{1}}, 0\right)$, which exists for $\mu_{1}>0$ and is unstable;

(c) $\mathrm{RW}_{\mathrm{S}}=\left(\mu_{2} / \chi+O\left(\mu_{2}^{2}\right), \sqrt{\sigma\left(\mu_{1}-\mu_{2}^{2} / \chi^{2}\right)+O\left(\mu_{2}^{3}\right)}\right)$, which exists for $\sigma\left(\mu_{1}-\right.$ $\left.\mu_{2}^{2} / \chi^{2}\right)>0$ and is stable iff $\sigma \chi<0$ and $\mu_{2} \chi<0$.

The normal form (6.1) admits distinct dynamic scenarios, depending on the values of $\chi$ and $\sigma$. A comprehensive description of these scenarios is given in Kuznetsov (1998). Since the periodic solution RWS in our flow is stable and exists for $\mu_{2}>0$, then $\sigma=+1$. For this scenario, the bifurcation diagram and corresponding phase portraits in the neighbourhood of the fold-Hopf point where $\mathrm{SN}_{2}$ and $\mathrm{H}_{2}$ coincide are presented in figure 11. For $\sigma \chi<0$, complex solutions exist in the neighbourhood of the fold-Hopf bifurcation point, including two-tori, heteroclinic structures, homoclinic solutions and more. The normal form (6.1) is generic in the sense that no symmetry considerations were imposed in its derivation. Although our system has $S O(2) \times Z_{2}$ symmetry, this does not alter the normal form in our case since the $S O(2)$ group does not affect the normal form (see Iooss \& Adelmeyer 1998), and for the region in parameter space under investigation, the $Z_{2}$ symmetry is not broken (the steady state $\mathrm{s}_{2}$ is point-wise $Z_{2}$ symmetric and the rotating wave RWS is set-wise $Z_{2}$ symmetric).

The phase portraits in figure 11 are projections onto $(x, \rho)$; rotation about the horizontal axis $x$ recovers angle $(\phi)$ information. The $x$-axis is the axisymmetricinvariant subspace. The fixed points on the $x$-axis $\left(s_{2}\right.$ and $\left.s_{M}\right)$ correspond to steady 
axisymmetric states. The off-axis fixed point corresponds to a limit cycle (the rotating wave $\mathrm{RWS}_{\mathrm{S}}$ ). The limit cycle in region 5 is an unstable modulated rotating wave MRW. The parametric portrait in the centre of the figure consists of seven distinct regions separated by different bifurcation curves. Initial conditions starting in region $1\left(\mu_{1}<0\right)$ evolve to far away states, not related to the fold-Hopf bifurcation (in our system, they evolve towards the $\mathrm{s}_{1}$ steady state). As $\mu_{1}$ changes sign for $\mu_{2}>0$, the saddle-node bifurcation curve $\mathrm{SN}_{2}$ is crossed and a pair of fixed points appears: $\mathrm{s}_{2}$ stable and $\mathrm{S}_{\mathrm{M}}$ unstable (region 2); the basin of attraction of $\mathrm{S}_{2}$ is shown shaded in the figure. On further increasing $\mu_{1}$, the stable fixed point $\mathrm{s}_{2}$ undergoes a supercritical Hopf bifurcation $\left(\mathrm{H}_{2}\right)$, becomes unstable and a limit cycle (the rotating wave $\mathrm{RW}_{\mathrm{S}}$ ) emerges (region 3); its basin of attraction is shown shaded in the figure. If we continue increasing $\mu_{1}$, according to the analysis of the normal form (6.1), a heteroclinic invariant two-dimensional manifold appears, as a result of the stable and unstable manifolds of $s_{2}$ and $s_{M}$ coinciding (the thick line in the phase portrait 4). The basin of attraction of $\mathrm{RW}_{\mathrm{S}}$ (shaded region) is delimited by the heteroclinic connection. This connection occurs along the straight line in the middle of region 4. However, this invariant sphere is a highly degenerate heteroclinic structure and high-order terms in the normal form destroy it (see discussions in Wiggins 1988; Guckenheimer \& Holmes 1983; Kuznetsov 1998). In a generic system, instead of a single bifurcation curve associated with this invariant sphere, there is a horn-shaped region about it (the hatched region 4). Generically, the unstable invariant manifold of $s_{2}$ and the stable invariant manifold of $S_{M}$ intersect transversally. This transversal intersection begins and ends in two heteroclinic tangency curves which are the limiting curves of the horn region 4. Inside region 4, the dynamics can be extremely complex, including an infinity of two-tori, solutions homoclinic and heteroclinic to both unstable fixed points, cascades of saddle-node and period-doubling bifurcations, and chaos. However, in this fold-Hopf scenario, the only local stable solution of the system is $\mathrm{RW}_{\mathrm{S}}$ and the complex dynamics associated with the horn are transient. On leaving the horn region (crossing the line 4 in the normal form), an unstable two-torus MRW appears from a heteroclinic bifurcation between $\mathrm{s}_{1}$ and $\mathrm{s}_{\mathrm{M}}$. The two-torus surrounds $\mathrm{RW}_{\mathrm{S}}$, and the basin of attraction of $\mathrm{RW}_{\mathrm{S}}$ is the interior of the two-torus. On decreasing $\mu_{2}$ in region 5, the two-torus MRW collapses in on $\mathrm{RW}_{\mathrm{S}}$ at a subcritical Neimark-Sacker bifurcation $\mathrm{NS}_{2}$. On further decreasing $\mu_{2}$ into regions 6 and 7, all local equilibria are unstable and trajectories evolve away from these regions of phase space to $s_{1}$.

Both the experiments and the numerical simulations find that on decreasing $\Gamma$ for fixed $R e$ in the range $R e \in(400,700)$, RW loses stability at a subcritical NeimarkSacker bifurcation, $\mathrm{NS}_{2}$ (shown in figure 8 as open and filled squares for the experimentally and numerically determined curve, respectively). Since no stable solution arises from the subcritical $\mathrm{NS}_{2}$, the resulting modulated rotating wave only occurs as a transient. Figure 12 shows a time series of the low-pass-filtered LDV measurements of the axial velocity at $z=0$ and $r=r_{i}+1.5 \mathrm{~mm}$ (LDV provides a voltage which is proportional to the Doppler-shift of the light scattered by small latex particle suspended in the flow, which is proportional to the velocity in the direction of view), that was recorded experimentally after $R e$ had been slightly increased from a value where RWS was stable to $R e=405$, at $\Gamma=2.94$, i.e. from region 5 to region 6 in figure 11. It shows the transient response of the flow to a (small) increase in $R e$. Since the precession frequency of the RWS is filtered out by the low-pass filter with cut-off frequency $f=0.2 \mathrm{~Hz}$, the oscillatory response corresponds to the modulation frequency of the unstable modulated rotating wave. The low modulation frequency provides experimental evidence that the Neimark-Sacker bifurcation curve has been 


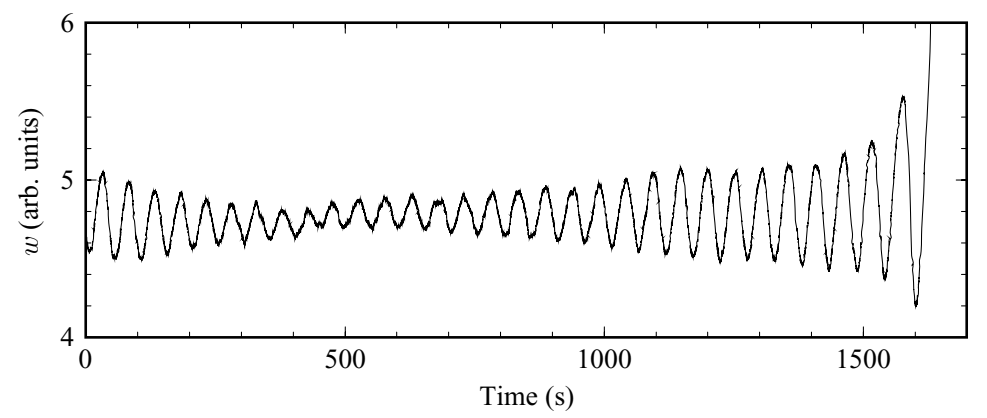

Figure 12. Time series of axial velocity $w$ recorded in the axial midplane at a distance of $1.5 \mathrm{~mm}$ from the inner cylinder and low-pass filtered with a cut-off frequency $f=0.2 \mathrm{~Hz}$. The low-frequency component of a transient modulated wave at $\Gamma=2.94$ and $R e=405$ is depicted. The end of the time series indicates the transition to $\mathrm{s}_{1}$. The viscous time is $d^{2} / v \approx 15.3 \mathrm{~s}$.

crossed. Since the bifurcation is subcritical, this modulated flow state occurs only as a transient. The end of the time series shown in figure 12 indicates the transition from a transient modulated wave to the far-off state $\mathrm{s}_{1}$.

In the region between the $\mathrm{NS}_{2}$ and $\mathrm{H}_{2}$ curves, $\mathrm{RW}_{\mathrm{S}}$ is the only locally stable state ( $\mathrm{s}_{1}$ is also stable, but it is 'far away' in phase space from the equilibria associated with the $\mathrm{s}_{2}$ solution manifold), until Re is larger than about 640. In the following section, the stability of $\mathrm{RW}_{\mathrm{S}}$ for $R e>640$ is explored.

\section{Instabilities of the $Z_{2}$-symmetric rotating wave $\mathrm{RW}_{\mathrm{S}}$}

The codimension-one bifurcations that a $Z_{2}$-symmetric limit cycle can undergo correspond to either a real Floquet multiplier $\mu=+1$ or a pair of complex-conjugate Floquet multipliers $\mu=\mathrm{e}^{ \pm \mathrm{i} \omega}$ crossing the unit circle. The period-doubling bifurcation with $\mu=-1$ is inhibited due to the presence of the $Z_{2}$ symmetry (Swift \& Wiesenfeld 1984). When $Z_{2}$ symmetry is broken, the $\mu=+1$ bifurcation is a pitchfork of limit cycles (cyclic pitchfork, CP), which can be either super- or subcritical, and a pair of (symmetrically related) non-symmetric limit cycles is born. If the $Z_{2}$ symmetry is preserved, the $\mu=+1$ bifurcation is a saddle-node bifurcation of cycles (cyclic fold, $\mathrm{CF})$. In the Neimark-Sacker bifurcation, $\mathrm{NS}_{\mathrm{s}}$ with $\mu=\mathrm{e}^{\mathrm{\pm} \mathrm{i} \omega}$, a modulated rotating wave is born; this quasi-periodic solution evolves on a two-torus, $T_{s}^{2}$, which is $Z_{2}$ symmetric although the individual quasi-periodic solutions on it are not (Kuznetsov 1998).

The $Z_{2}$-symmetric limit cycle in the present Taylor-Couette flow, RWS, undergoes two of the aforementioned bifurcations: for $\Gamma>3.18, Z_{2}$ symmetry is broken and a pair of asymmetric limit cycles, $\mathrm{RW}_{\mathrm{A}}$, appear in a cyclic pitchfork bifurcation; for $\Gamma<3.18$ a Neimark-Sacker bifurcation takes place, resulting in a $Z_{2}$-symmetric two-torus $T_{s}^{2}$ with quasi-periodic solutions MRWs. The loci of these two bifurcation curves are plotted in figure 13 where their numerically and experimentally determined locations in $(\Gamma, R e)$ space are shown with filled and open circles respectively. Also shown in the figure are bifurcation curves with $\Gamma \sim 3.16$ that divide the parameter space into regions where MRWS and $\mathrm{RW}_{\mathrm{A}}$ exist; these will be described and discussed in $\S 8$. 


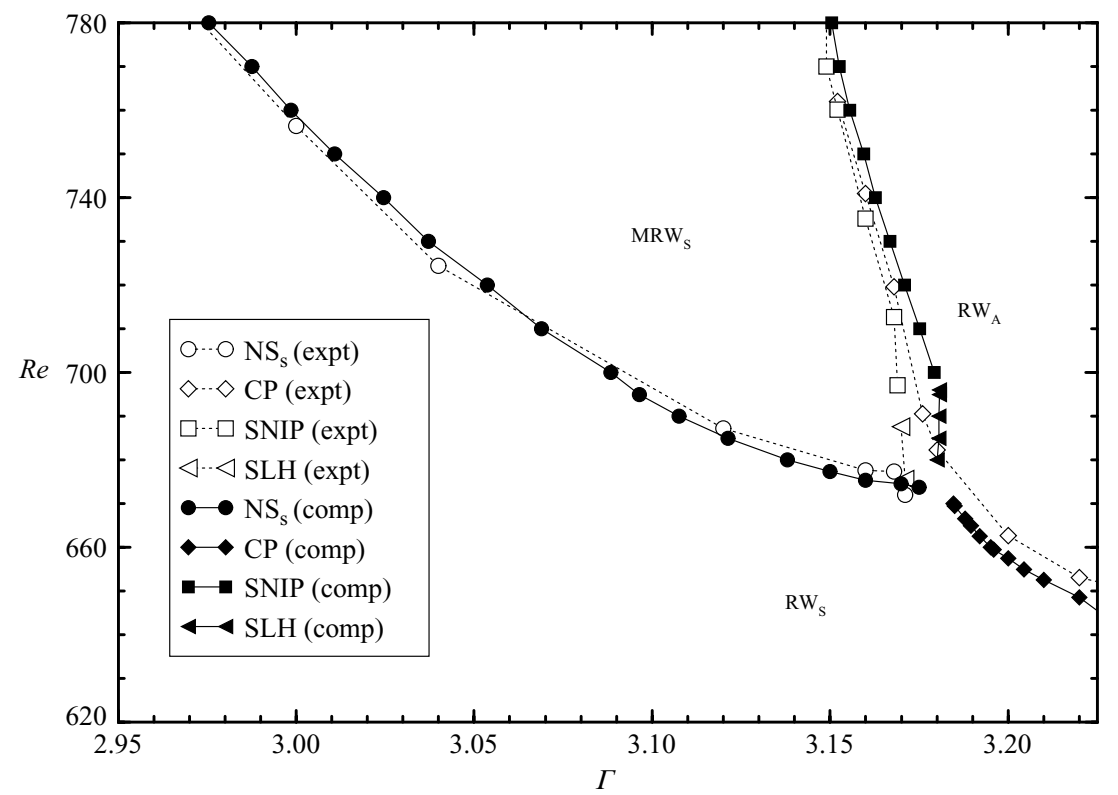

FIGURE 13. Bifurcation curves in $(R e, \Gamma)$ space: $\mathrm{NS}_{\mathrm{s}}$ is the curve of Neimark-Sacker bifurcations at which the RWS becomes unstable and spawns the $Z_{2}$-symmetric $T_{s}^{2}$ with MRWS solutions; $\mathrm{CP}$ is the curve of cyclic pitchfork bifurcations at which RW becomes unstable and a pair of $Z_{2}$-conjugate $\mathrm{RW}_{\mathrm{A}}$ is spawned; SNIP is the saddle-node-infinite-period bifurcation curve on which saddle-nodes of $\mathrm{RW}_{\mathrm{A}}$ occur upon $T_{s}^{2}$; SLH is a curve of saddle-loop homoclinic bifurcations at which $\mathrm{MRW}_{\mathrm{S}}$ becomes heteroclinic to a pair of saddle $Z_{2}$-conjugate rotating waves. Curves with open (filled) symbols are determined experimentally (numerically).

\subsection{Neimark-Sacker bifurcation of $\mathrm{RW}_{\mathrm{S}}$}

The Neimark-Sacker bifurcation $\mathrm{NS}_{\mathrm{s}}$ of $\mathrm{RW}_{\mathrm{S}}$ occurs for $\Gamma \lesssim 3.17$ and a modulated rotating wave MRW is spawned. For rotating waves, the modal kinetic energies $E_{m}$ are constant in time, and for modulated rotating waves, $E_{m}$ are time-periodic. The amplitude and period of the oscillations in $E_{1}$ for $\mathrm{MRW}_{\mathrm{S}}$ are global measures of the modulation amplitude squared (the kinetic energy is a squared quantity) and modulation period of MRWS, denoted $\Delta E_{1}$ and $\tau_{2}$, respectively.

Figure 14 shows the variation with $R e$ of $(a) \Delta E_{1}$ and $(b) \tau_{2}$ of MRW for various $\Gamma$. Note that for the smaller values of $\Gamma(\lesssim 3.15), \Delta E_{m}$ grows linearly with $R e$ and the modulation period $\tau_{2}$ varies very slowly with $R e$, indicative of the supercritical nature of the Neimark-Sacker bifurcation. However, for $\Gamma=3.16$, the figures show that the nature of the bifurcation changes; the amplitude loses its linearity very soon after the bifurcation, and the period varies significantly at onset. In fact, the figure suggests that the period is becoming unbounded at onset. Figure 15 shows the modulation period near onset of the Neimark-Sacker bifurcation as a function of $\Gamma$; $\tau_{2}$ increases significantly as $\Gamma$ approaches 3.18, and follows an inverse square-root law, $\tau_{2} \sim 1 / \sqrt{\left|\Gamma-\Gamma_{c}\right|}$, as shown by the straight line fit.

Since MRW bifurcates from a $Z_{2}$-symmetric limit cycle $\mathrm{RW}_{\mathrm{S}}$, then the two-torus upon which MRWS evolves is also (set-wise) $Z_{2}$-symmetric (Kuznetsov 1998). The modal energies, $E_{m}$, being squared quantities, mask this symmetry; time series of $E_{m}$ for MRWS show a periodicity equal to $\tau_{2} / 2$. In order to reveal the symmetry, we plot in figure $16(a)$ a phase portrait of $\mathrm{MRW}_{\mathrm{S}}$ projected onto the plane $\left(W^{-}, W^{+}\right)$where $W^{ \pm}$ 

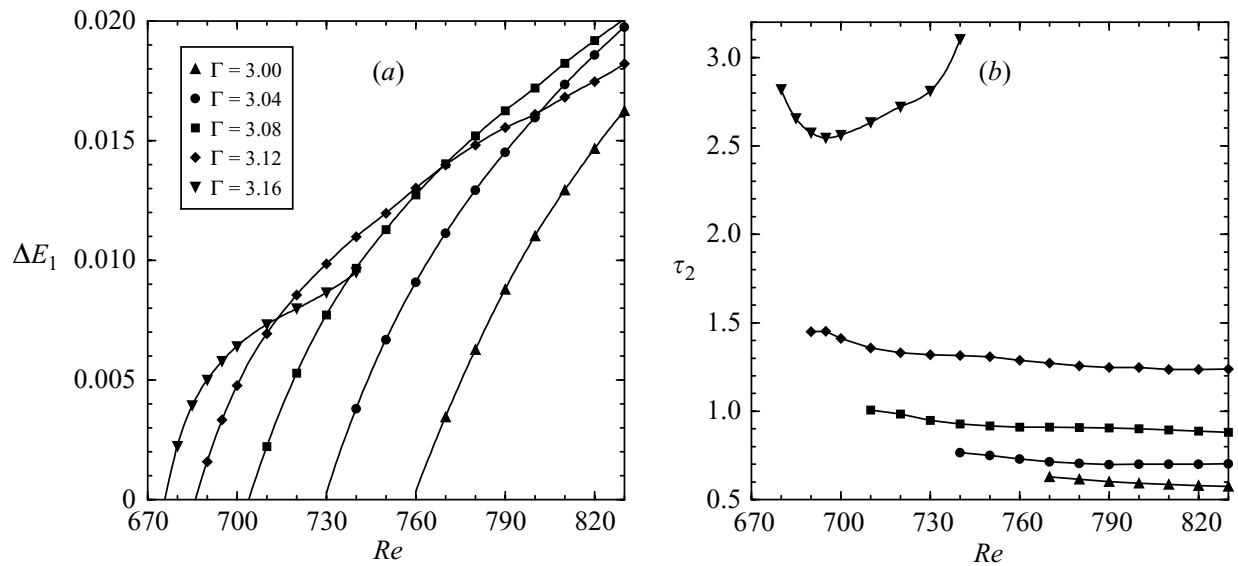

FIGURE 14. Variation with $R e$ of $(a)$ the modulation amplitude $\Delta E_{1}$ and $(b)$ the modulation period $\tau_{2}$ of MRW for various $\Gamma$ as indicated.
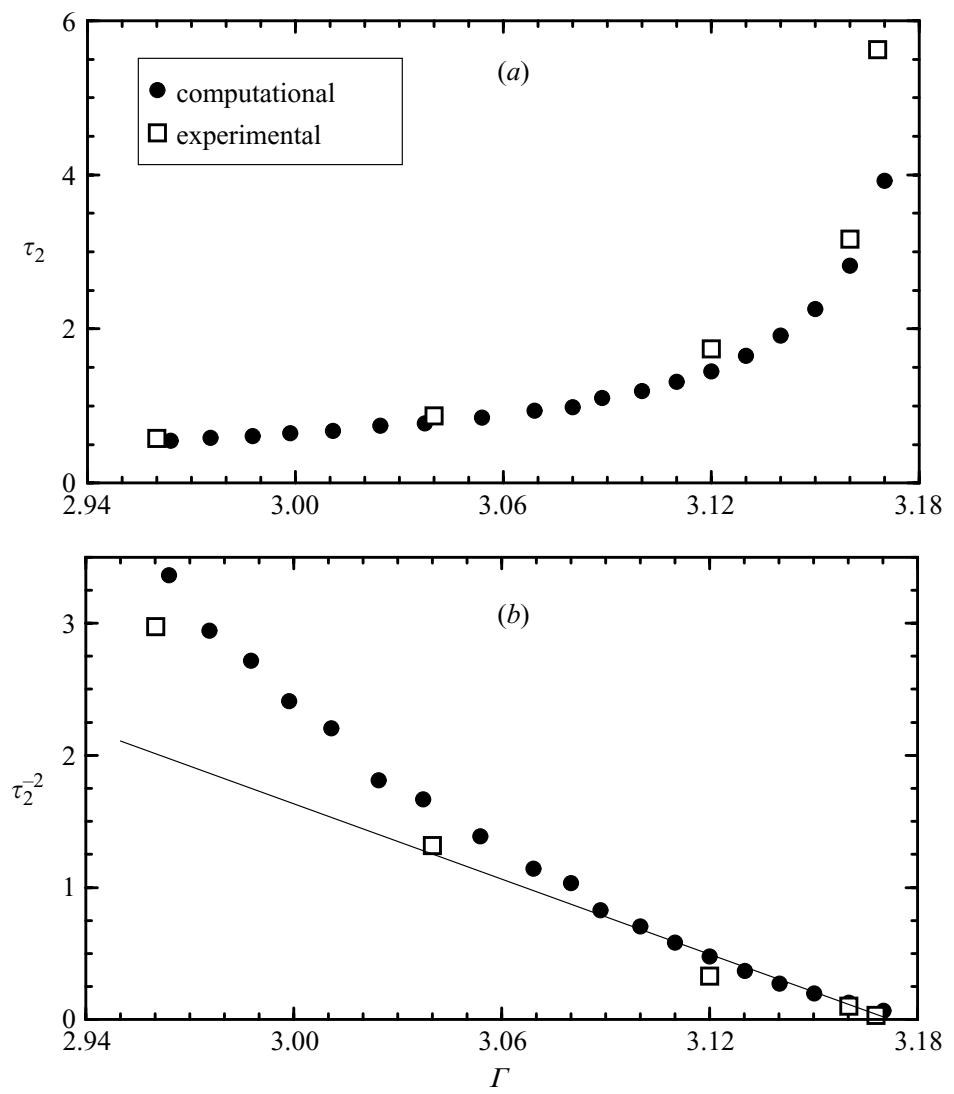

FIGURE 15. Variation with $\Gamma$ of the modulation period of MRWS, $\tau_{2}$, very close to the Neimark-Sacker bifurcation, in viscous time units $d^{2} / v$, determined numerically and experimentally. The straight line is a fit to the nine computed points with larger $\Gamma$. 

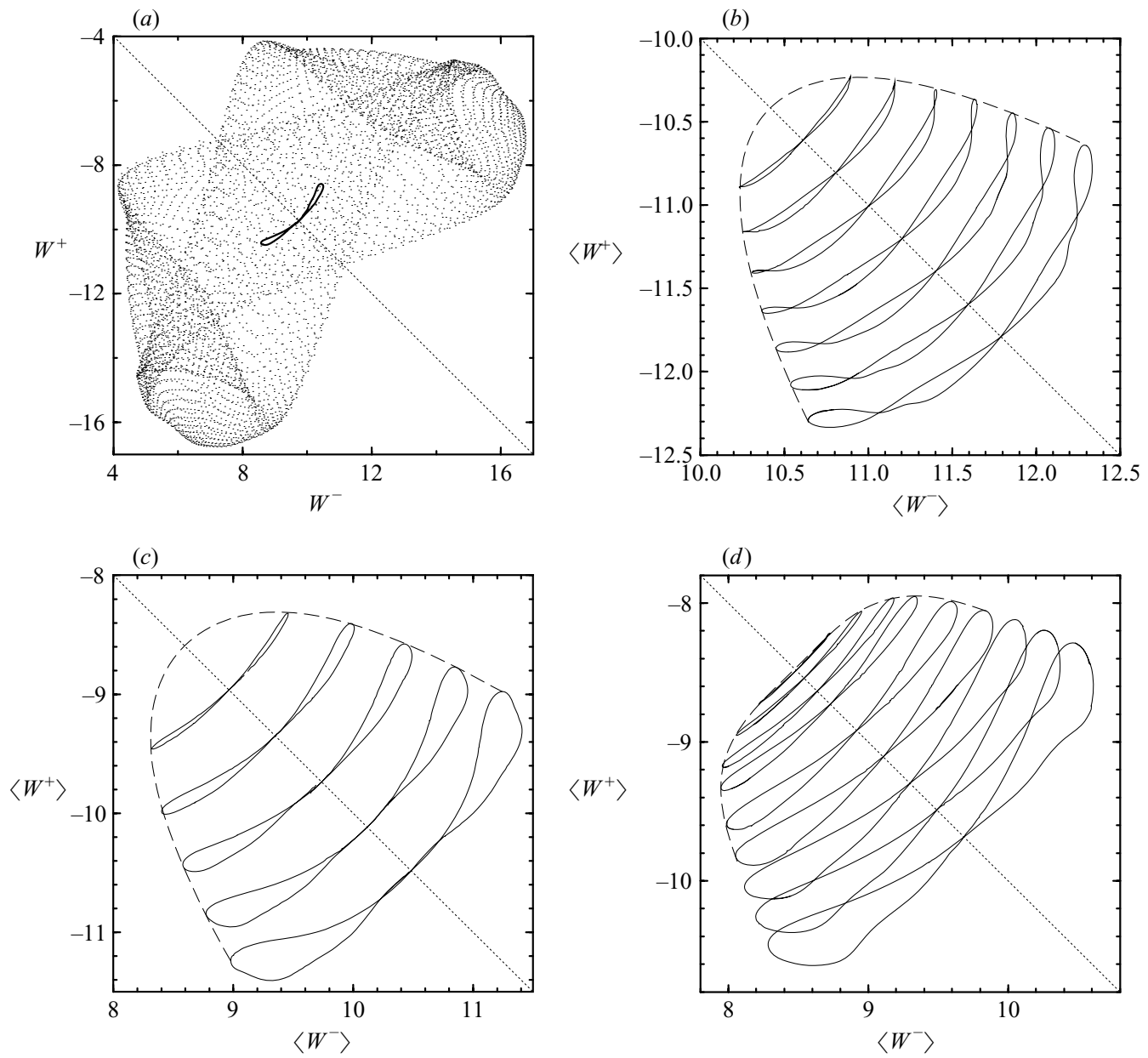

FIGURE 16. (a) Phase portrait $\left(W^{-}, W^{+}\right)$(dots) and filtered phase portrait $\left(\left\langle W^{-}\right\rangle,\left\langle W^{+}\right\rangle\right)$ (solid) of MRWS for $\Gamma=3.12, R e=740$. (b) Filtered phase portraits of MRW for $\Gamma=3.0$ and $R e=770$ to 830 in steps of 10 . (c) Same as $(b)$ for $\Gamma=3.12$ and $R e=700$ to 780 in steps of 20. (d) Same as $(b)$ for $\Gamma=3.16$ and $R e=677,680,685$ and 690 to 740 in steps of 10 . Dashed lines are quartic fits.

are the axial velocities at the points $\left(r=r_{i} / d+1 / 2, \theta=0, z= \pm \Gamma / 2\right)$. The reflection symmetry of the phase portrait about the line $W^{+}+W^{-}=0$ is indicative of the $Z_{2}$ symmetry of the two-torus. The two-torus looks rather convoluted; this is because the fast frequency (corresponding to the precession frequency of the underlying $\mathrm{RW}_{\mathrm{S}}$ from which MRW bifurcates) has large amplitude and the slow frequency (the modulation) has low amplitude since MRWS bifurcates supercritically. So, instead of the limit cycle 'shedding its skin' (which would give a two-torus that looks like a doughnut), in this case the limit cycle 'wobbles'. Now, the fast (precession) frequency $(2 \pi / \tau)$ is a constant (in time) that depends only weakly on parameters, and so the phase dynamics associated with the underlying rotating wave component of MRW are trivial and essentially decouple from the rest of the dynamics. This allows us to low-pass filter these solutions and extract a clearer picture of the dynamics. Given a function 

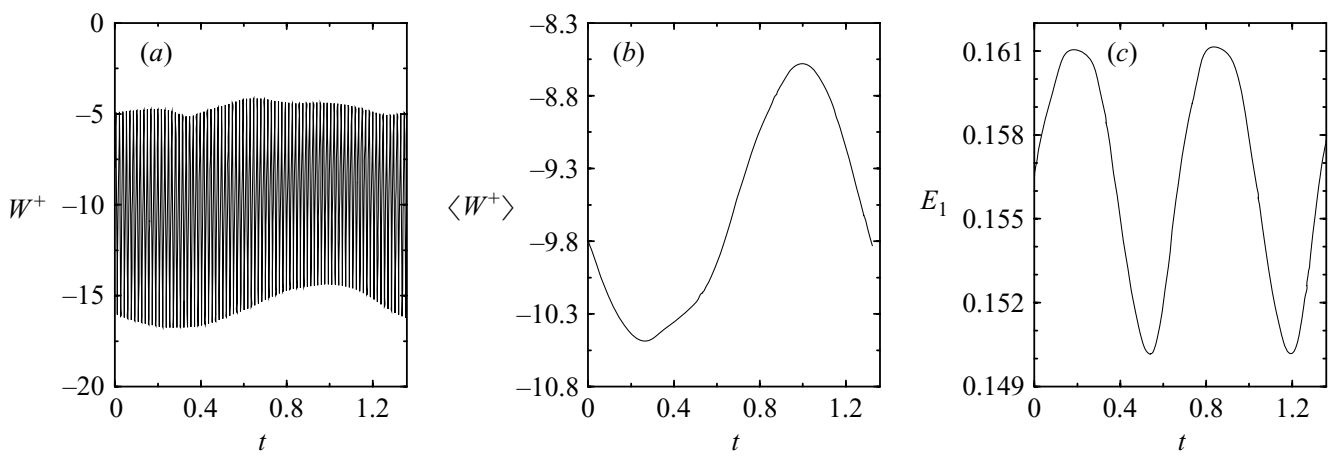

FIGURE 17. Time series of MRWs at $R e=740$ and $\Gamma=3.12$.

\begin{tabular}{ccrc}
$\Gamma$ & $a$ & \multicolumn{1}{c}{$b$} & $c$ \\
3.00 & 0.0998 & 0.950 & 14.73 \\
3.12 & 0.1076 & 0.563 & 12.15 \\
3.16 & 0.2735 & -0.014 & 11.98
\end{tabular}

TABLE 1. Quadratic fits to the amplitudes of the filtered portraits of MRWS shown in figure 16: $y=a x^{4}+b x^{2}+c$, where $x=-\left(\left\langle W^{+}\right\rangle+\left\langle W^{-}\right\rangle\right) / \sqrt{2}, y=-\left(\left\langle W^{+}\right\rangle-\left\langle W^{-}\right\rangle\right) / \sqrt{2}$.

of time $f(t)$, the filtered function, $\langle f(t)\rangle$, is a running average defined as

$$
\langle f(t)\rangle=\frac{1}{\tau} \int_{t-\tau / 2}^{t+\tau / 2} f(y) \mathrm{d} y
$$

where $\tau$ is the precession period associated with the rotating wave component of the solution considered. The modulation period, $\tau_{2}$, is considerably longer, and is not filtered out.

Figure 17 illustrates the low-pass filtering of a MRW solution at $R e=740$ and $\Gamma=3.12$. Part $(a)$ of the figure is the time series of the axial velocity at a point $W^{+}$ over approximately one modulation period (about 1.4 viscous times). This modulation period is much greater than the underlying precession period (about 0.03 viscous time) which can only just be resolved in the plot. Applying the low-pass filter results in the periodic signal $\left\langle W^{+}\right\rangle$shown in part $(b)$ of the figure. The corresponding time series of the modal energy $E_{1}$ is shown in part $(c)$ of the figure; both $\left\langle W^{+}\right\rangle$and $E_{1}$ are periodic (the fast oscillations in $W^{+}$are filtered out of $\left\langle W^{+}\right\rangle$, and since the fast oscillations correspond to a precession they do not appear in $E_{1}$ ). The $Z_{2}$ symmetry of MRW is responsible for the period of $E_{1}$ being half of that of $\left\langle W^{+}\right\rangle$.

Figure $16(a)$ is a phase portrait of $\mathrm{MRW}_{\mathrm{S}}$ at $R e=740$ and $\Gamma=3.12$ projected onto $\left(W^{-}, W^{+}\right)$(dots) as well as its low-pass filter projected onto $\left(\left\langle W^{-}\right\rangle,\left\langle W^{+}\right\rangle\right)$(solid curve). The filtered portrait is a limit cycle of much smaller extent than the unfiltered portrait (the two-torus). The extent of the limit cycle is another measure of the modulation amplitude. In parts $(b),(c)$ and $(d)$ of figure 16 are filtered portraits of MRWS at $\Gamma=3.00,3.12$ and 3.16, respectively, for a number of $R e$ values. The filtered phase portraits of $\mathrm{RW}_{\mathrm{S}}$ from which the $\mathrm{MRW}_{\mathrm{S}}$ bifurcate are points that lie on the line $\left\langle W^{+}\right\rangle=-\left\langle W^{-}\right\rangle$. The dashed curves enveloping the MRWS filtered portraits are curve fits of the form $y=a x^{4}+b x^{2}+c$, where $x=-\left(\left\langle W^{+}\right\rangle+\left\langle W^{-}\right\rangle\right) / \sqrt{2}$ and $y=-\left(\left\langle W^{+}\right\rangle-\left\langle W^{-}\right\rangle\right) / \sqrt{2}$. The values of the fitted coefficients are listed in table 1 . The 


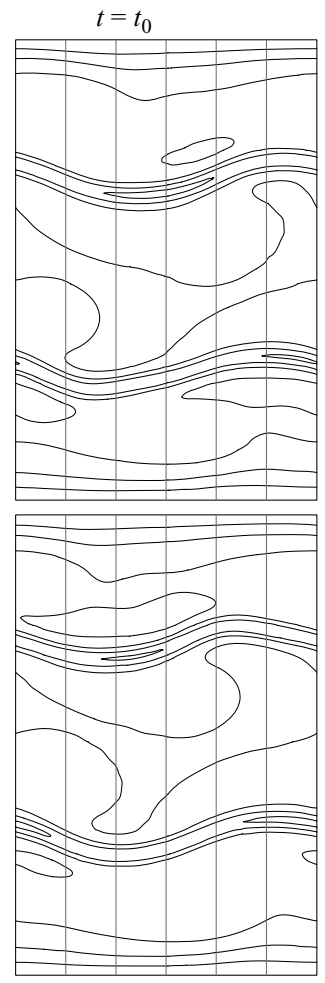

$t=t_{0}+40 \tau$
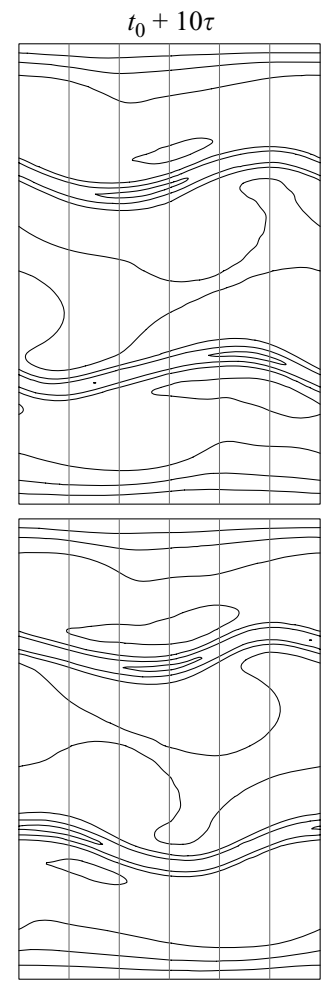

$t_{0}+50 \tau$
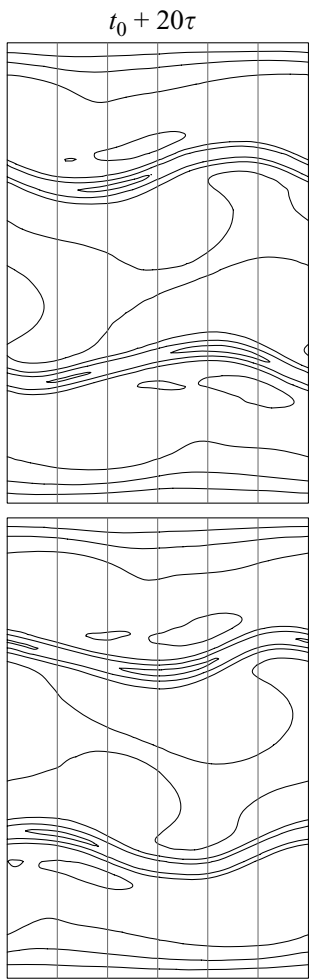

$t_{0}+60 \tau$

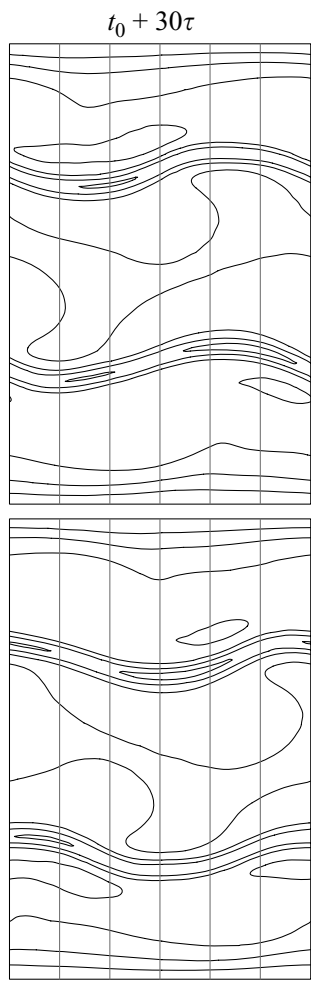

$t_{0}+70 \tau$

FIGURE 18. Contours of $v$ in the cylindrical plane $r=r_{i} / d+0.3$ of $\mathrm{MRW}_{\mathrm{S}}$ at $R e=740$, $\Gamma=3.12, \eta=0.5$. The sequence covers (approximately) one modulation period $\tau_{2}$, and the frames are ten precession periods apart $(10 \tau)$. There are twelve equispaced contours with the levels $v \in[0, R e]$. Vertical lines at angles $\theta=j \pi / 3$ for $j=1$ to 5 are included.

fit for $\Gamma=3.00$, and to a slightly lesser degree for $\Gamma=3.12$, is quadratic, typical of a supercritical Neimark-Sacker bifurcation. For $\Gamma=3.16$ however, the quartic term dominates and we find that the Neimark-Sacker bifurcation is slightly subcritical.

The Floquet multipliers for the Neimark-Sacker bifurcation are complex conjugates of the form $\mu=\exp ( \pm \mathrm{i} \alpha)$, and the modulation period is of the form $\tau_{2}=(2 \pi / \alpha) \tau$, where $\tau$ is the period of the limit cycle from which the quasi-periodic solution bifurcates. For MRW , we have shown (figure 15) that as $\Gamma$ increases beyond about $3.15, \tau_{2} \rightarrow \infty$ so that $\alpha \rightarrow 0$ and $\mu \rightarrow+1$. So, not only is the Neimark-Sacker bifurcation becoming subcritical, it is also becoming degenerate as $\Gamma \rightarrow 3.18$.

Figure 18 shows the jet dynamics associated with the modulation period $\tau_{2}$, for MRW $_{S}$ at $R e=740, \Gamma=3.12, \eta=0.5$; this is the same MRW as shown in figures $16(a)$ and 17. The figure shows contours of $v$ in the cylindrical plane $r=r_{i} / d+0.3$, strobed every ten precession periods; in fact we have computed Poincare sections of MRWS and recorded the velocity field every time it crosses the section given by $U^{+}=-20.0$ (where $U^{+}$is the radial velocity at the point $r=r_{i} / d+1 / 2, \theta=0, z=\Gamma / 2$ ). The modulation period is $\tau_{2}=1.31$, while the precession period is $\tau=0.0164$; there are $\tau_{2} / \tau=80.3$ precession periods in every modulation period. Figure 18 consists of eight frames that are 10 precession periods apart, and hence it very nearly covers one modulation period $\tau_{2}$. In the three first frames the upper jet phase (position of the 

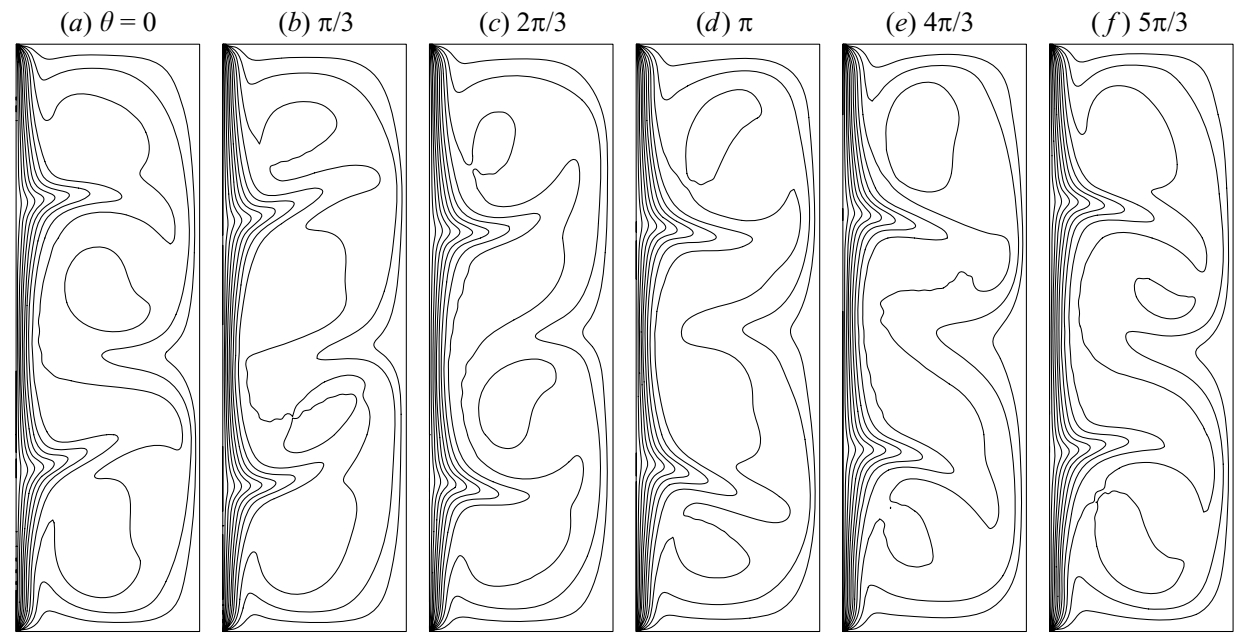

FIGURE 19. Contours of $v$ in various meridional plane, as indicated, for $\mathrm{RW}_{\mathrm{A}}$ at $R e=700$, $\Gamma=3.20, \eta=0.5$; computed with $N_{z}=96, N_{r}=48, N_{\theta}=20, \delta t=2 \times 10^{-5}$. There are twelve positive contours with the levels $v \in[0, R e]$.

maxima and minima) is advanced in $\theta$ with respect to the lower jet. In frames five to seven, the opposite is true. In frames 4 and 8 the two jets are almost in phase. Therefore, the dynamics associated with the Neimark-Sacker bifurcation correspond to an oscillation of the relative phase between the upper and lower jets, with period $\tau_{2}$. The flow is no longer $Z_{2}$-symmetric, but the two-tori on which it evolves are $Z_{2}$ symmetric. This is clearly seen in figure 18: the frames in the second row are almost exactly the axial reflection $(z \rightarrow-z)$ of the corresponding frames in the first row, with an appropriate rotation in the azimuthal direction. But the quasi-periodic character of the solution (it never repeats itself if the frequencies are incommensurate) prevents an individual MRWS solution from having an exact spatio-temporal $Z_{2}$ symmetry.

\subsection{Cyclic pitchfork bifurcation of $\mathrm{RW}_{\mathrm{S}}$}

We now turn our attention to the instability of $\mathrm{RW}_{\mathrm{S}}$ for $\Gamma>3.18$, which is due to a pitchfork bifurcation breaking $Z_{2}$ symmetry. Figure 19 shows contours of the azimuthal velocity $v$ of one of the pair of bifurcating rotating waves $\mathrm{RW}_{\mathrm{A}}$, which has azimuthal wavenumber $m=1$, at $R e=700$ and $\Gamma=3.20$ in various meridional planes. Figure 20 shows the same contours on a cylindrical surface $(\theta, z)$ at $r=$ $r_{i} / d+0.3$, projected on a plane and compressed in azimuth by a factor of four in order to better display the structure of the tilt wave. The lack of reflection symmetry about $z=0$ is immediately obvious (as it was for $\mathrm{RW}_{\mathrm{S}}$ ), but unlike $\mathrm{RW}_{\mathrm{S}}, \mathrm{RW}_{\mathrm{A}}$ is not invariant to a reflection composed with a rotation through $\pi$. In essence, the $Z_{2}$ symmetry breaking corresponds to the two jets emerging from the inner cylinder boundary layer being tilted out of phase with respect to each other (the tilts are in-phase for RWS). From the figure, one can see that the phase difference is about $2 \pi / 9\left(40^{\circ}\right)$ for this case. The corresponding $Z_{2}$-conjugate $\mathrm{RW}_{\mathrm{A}}$ is the reflection of $\mathrm{RW}_{\mathrm{A}}$ through $z=0$, so that the phase difference between the tilts of the top and bottom jets is the negative of that of its partner.

To obtain a quantitative measure of the breaking of $Z_{2}$ symmetry (consisting of the combination of the $z$-reflection and an azimuthal rotation of $\pi / m$ ) of $\mathrm{RW}_{\mathrm{A}}$ (which 
(a)

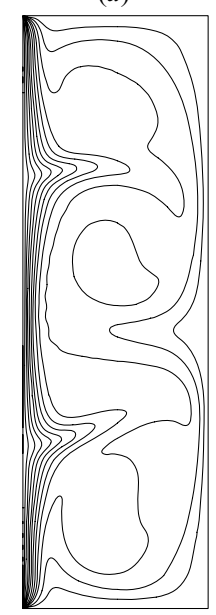

(b)

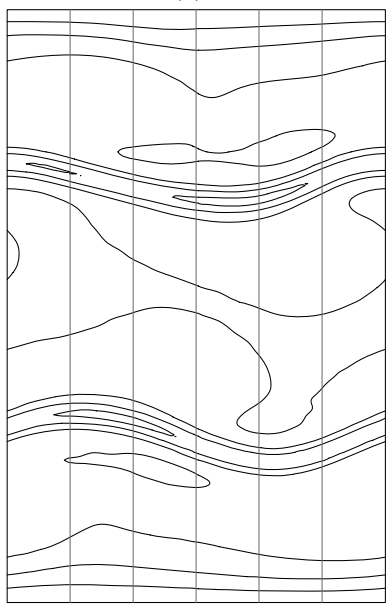

(c)

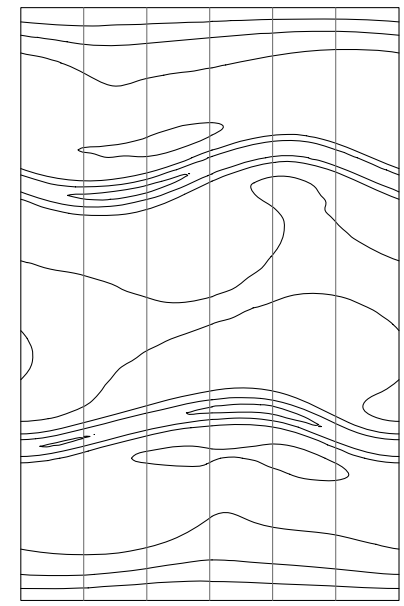

Figure 20. Contours of $v$ in $(a)$ the meridional plane $\theta=0$, and $(b)$ the cylindrical projection $(\theta, z)$ at $r=r_{i} / d+0.3$, for $\mathrm{RW}_{\mathrm{A}}$ at $\operatorname{Re}=700, \Gamma=3.2, \eta=0.5$. Vertical lines at angles $\theta=j \pi / 3$ for $j=1$ to 5 are included in $(b)$, which correspond to the meridional planes used in figure 19 . There are twelve equispaced contours with the levels $v \in[0, R e]$. (c) The $Z_{2}$-conjugate state of $(b)$.
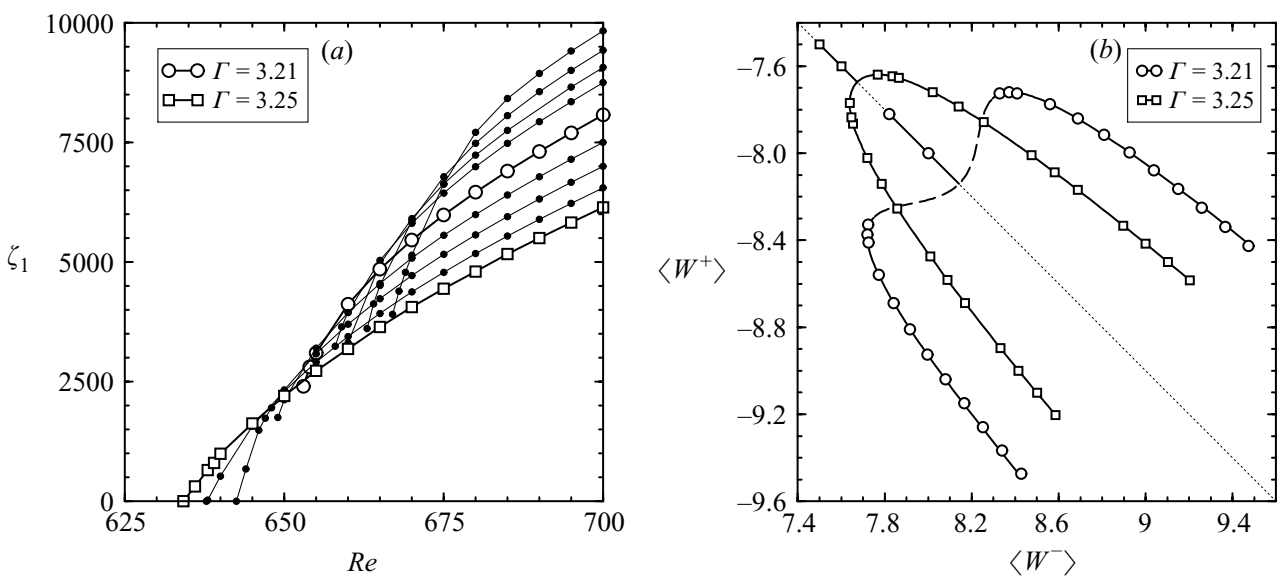

FIgURE 21. (a) Variation of $\zeta_{1}$ for $\mathrm{RW}_{\mathrm{A}}$ with $\operatorname{Re}$ for $\Gamma=3.188,3.192,3.196$ and 3.20 to 3.25 in steps of 0.01. (b) Filtered phase portraits of $\mathrm{RW}_{\mathrm{S}}$ and $\mathrm{RW}_{\mathrm{A}}$ at $\Gamma=3.21$ and 3.25 and for $R e$ values corresponding to the respective $\Gamma$ curves in $(a)$. Symbols correspond to computed solutions and lines are quartic fits.

has $m=1$ ), we introduce a parameter $\zeta_{m}$ :

$$
\zeta_{m}=\sum_{j=0}^{N_{r}} \sum_{k=-N_{\theta}}^{N_{\theta}}\left[\sum_{i=0}^{N_{z} / 2}\left|w_{2 i, j, k}\right|^{2} \cos ^{2}\left(\frac{k \pi}{2 m}\right)+\sum_{i=0}^{\left(N_{z}-1\right) / 2}\left|w_{2 i+1, j, k}\right|^{2} \sin ^{2}\left(\frac{k \pi}{2 m}\right)\right] .
$$

This parameter is zero when the rotating wave is $Z_{2}$ symmetric. $\zeta_{m}$ is a global measure of the velocity squared, and so at a supercritical pitchfork bifurcation, $\zeta_{m}$ of $\mathrm{RW}_{\mathrm{A}}$ should grow linearly with distance in parameter space from the bifurcation curve.

Figure 21(a) shows the variation with $R e$ of $\zeta_{1}$ for $\mathrm{RW}_{\mathrm{A}}$ at various $\Gamma$, from $\Gamma=3.188$ to 3.25 . For $\Gamma=3.23,3.24$ and 3.25 , we have been able to continue 


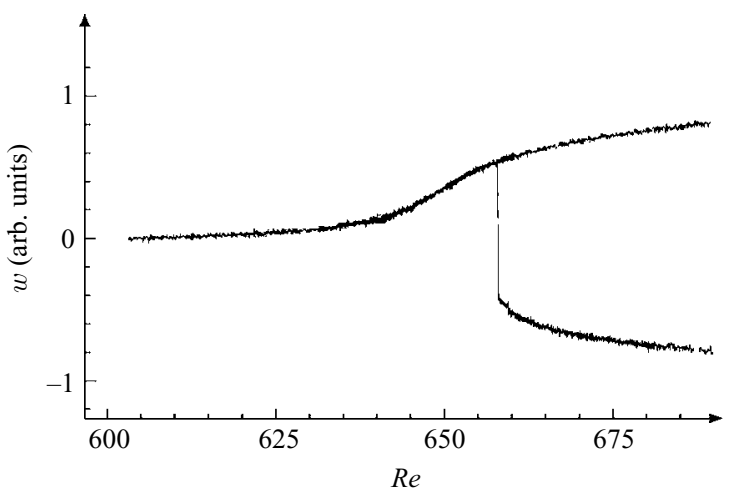

FIGURE 22. Measurement of axial velocity $w$ in the axial midplane showing an imperfect pitchfork bifurcation in the experiments at $\Gamma=3.208$. Lower border of decoupled branch provides a measure of the location of the fold bifurcation.

$\mathrm{RW}_{\mathrm{A}}$ solutions with decreasing $R e$ all the way to the pitchfork bifurcation where $\zeta_{1} \rightarrow 0$. However, for $\Gamma \leqslant 3.22$, below a certain value of $\operatorname{Re}$ (dependent on $\Gamma$ ) the flow started with an initial condition corresponding to $\mathrm{RW}_{\mathrm{A}}$ with finite $\zeta_{1}$ at a slightly larger $R e$ evolves to $\mathrm{RW}_{\mathrm{S}}$. This behaviour is indicative of a saddle-node (cyclic fold, $\mathrm{CF}$ ) bifurcation of $\mathrm{RW}_{\mathrm{A}}$, and hence that the cyclic pitchfork bifurcation is subcritical for $\Gamma \leqslant 3.22$. In figure $21(b)$, we plot the filtered phase portraits of $\mathrm{RW}_{\mathrm{S}}$ and $\mathrm{RW}_{\mathrm{A}}$ at $\Gamma=3.21$ and 3.25 for a range of $R e$ (roughly between 620 and 700 ). The low-pass filtering results in these phase portraits of rotating waves simply being fixed points. For RWS, the phase portraits are fixed points on the line $\left\langle W^{+}\right\rangle=-\left\langle W^{-}\right\rangle$, and for $\mathrm{RW}_{\mathrm{A}}$ and its $Z_{2}$-conjugate the portraits are a pair of points symmetric about this line. For $\Gamma=3.25$, the locus of phase portraits of $\mathrm{RW}_{\mathrm{A}}$ lies on a parabola about the line $\left\langle W^{+}\right\rangle=-\left\langle W^{-}\right\rangle$, giving the usual picture of a supercritical pitchfork bifurcation. For $\Gamma=3.21$, a quartic fit to the $\mathrm{RW}_{\mathrm{A}}$ points shows the typical form of a subcritical pitchfork bifurcation.

Due to unavoidable imperfections in the apparatus, the experiment does not have a perfect $Z_{2}$ symmetry and it is well known that imperfections may alter the structure of $Z_{2}$ symmetry-breaking bifurcations. Figure 22 depicts a bifurcation diagram of an imperfect cyclic pitchfork obtained experimentally at $\Gamma=3.208$. It is measured by a quasi-static variation of $R e$ while recording the axial velocity $w$ in the axial mid-plane at a radial distance of $1.5 \mathrm{~mm}$ from the inner cylinder. On increasing $R e$ from subcritical values, the nominally symmetric $\mathrm{RW}_{\mathrm{S}}$ is smoothly connected with an asymmetric $\mathrm{RW}_{\mathrm{A}}$. The conjugate decoupled asymmetric $\mathrm{RW}_{\mathrm{A}}$ (which is arrived at by sudden changes in $R e$ ) disappears catastrophically as $R e$ is decreased from supercritical values. The corresponding $R e$ is taken as a measure of the cyclic fold bifurcation of the imperfect cyclic pitchfork bifurcation. Here, the critical Reynolds number is estimated to be $R e_{c}=658.8$ for $\Gamma=3.208$.

The experimental loci of the cyclic pitchfork bifurcations, shown in figure 13 as open diamonds, correspond to estimates of the fold resulting from the imperfect pitchfork bifurcation. The SNIP (open squares) and saddle-loop homoclinic (open triangles) bifurcations, also shown in figure 13, are only found to occur on the smoothly connected asymmetric branch. These bifurcations in the experiment are therefore homoclinic instead of heteroclinic bifurcations in a perfectly symmetric flow (such as in the numerics). The two experimental bifurcation lines shown, instead of 


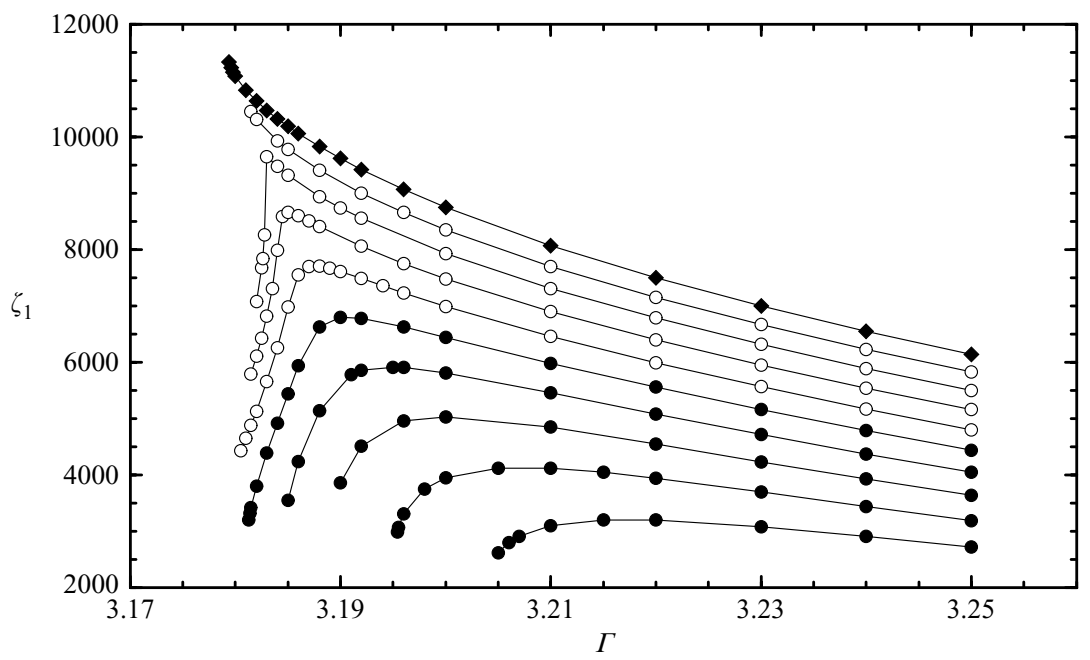

FIGURE 23. Variation of $\zeta_{1}$ for $\mathrm{RW}_{\mathrm{A}}$ with $\Gamma$ for $R e \in[655,700]$, in steps of 5 . For $R e \in[655,675]$ (filled circles), the $\mathrm{RW}_{\mathrm{A}}$ undergo cyclic fold (CF) bifurcations at low $\Gamma$. For $R e \in[680,695]$ (open circles), at low $\Gamma \mathrm{RW}_{\mathrm{A}}$ undergoes Neimark-Sacker $\left(\mathrm{NS}_{\mathrm{a}}\right)$ bifurcations spawning MRW $\mathrm{A}$. For $R e=700$ (filled diamonds), the $\mathrm{RW}_{\mathrm{A}}$ undergo a saddle-node-infinite-period (SNIP) bifurcation with another nearby saddle $\mathrm{RW}_{\mathrm{A}}$ (actually there is a pair of stable/saddle $\mathrm{RW}_{\mathrm{A}}$ due to the $Z_{2}$ symmetry). The heteroclinic connections between the $\mathrm{RW}_{\mathrm{A}}$ lead to the MRW following the SNIP bifurcation.

one in the numerics, are a consequence of the imperfection in the experimental system. The global bifurcations are described in detail in $\S 8$.

The $\zeta_{1}$ variation with $\Gamma$ of $\mathrm{RW}_{\mathrm{A}}$ is shown in figure 23 for $R e$ between 655 and 700. For $\Gamma>3.22, \zeta_{1}$ increases linearly with $R e$, but for smaller $\Gamma$, the behaviour of $\zeta_{1}$ is quite varied. For $R e$ between 655 and 675 (the curves with filled circles), $\mathrm{RW}_{\mathrm{A}}$ terminates at a cyclic fold bifurcation as $\Gamma$ is reduced (as discussed above). For $R e$ between 680 and 695 (curves with open circles), as $\Gamma$ is reduced, $\mathrm{RW}_{\mathrm{A}}$ loses stability at a supercritical Neimark-Sacker bifurcation $\left(\mathrm{NS}_{\mathrm{a}}\right)$ at which a modulated rotating wave $\mathrm{MRW}_{\mathrm{A}}$ is spawned (there are a pair of $Z_{2}$-conjugate $\mathrm{MRW}_{\mathrm{A}}$ bifurcating from the pair of $\mathrm{RW}_{\mathrm{A}}$ ). For $R e=700$ and higher, instead of suffering the local $\mathrm{NS}_{\mathrm{a}}$ bifurcation, $\mathrm{RW}_{\mathrm{A}}$ suffers a global bifurcation as $\Gamma$ is reduced. In the following section we explore the nature of this global bifurcation as well as the fate of $\mathrm{MRW}_{\mathrm{A}}$.

\section{8. $Z_{2}$ symmetry breaking via global bifurcations}

Across a curve along $\Gamma \sim 3.18$ (see figure 13, which shows both the numerically and the experimentally determined curve), a complex symmetry-breaking bifurcation process takes place providing a connection between the $Z_{2}$-symmetric state (MRWS) and the non- $Z_{2}$-symmetric states (either $\mathrm{RW}_{\mathrm{A}}$ or $\mathrm{MRW}_{\mathrm{A}}$ ). For the connection between $\mathrm{MRW}_{\mathrm{S}}$ and $\mathrm{RW}_{\mathrm{A}}$, this complex process involves a global SNIP (saddle-node-infiniteperiod) bifurcation with $Z_{2}$ symmetry.

The SNIP bifurcation consists of a saddle-node bifurcation taking place on a limit cycle, as shown schematically in figure 24(a). Before the bifurcation (left diagram), the period of the limit cycle tends to infinity as the bifurcation is approached, and the periodic solution spends more and more time near the place where the saddle-node will appear. The periodic solution becomes a homoclinic orbit at the bifurcation point 
(a)
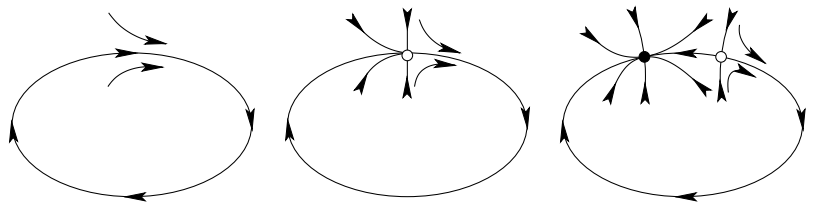

(b)
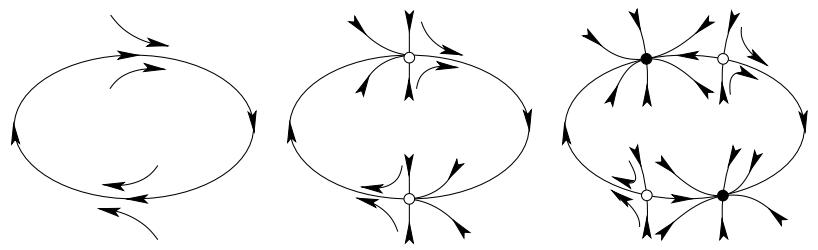

(c)

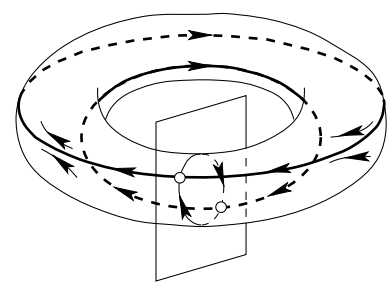

FIGURE 24. Schematic of the SNIP bifurcation on an invariant circle in $(a)$ a generic system, and $(b)$ a $Z_{2}$-symmetric system; left, central and right diagrams correspond to before, during and after the SNIP bifurcation. (c) Schematic of the SNIP bifurcation on an invariant two-torus; the planar section (a Poincaré section) corresponds to case $(b)$ centre.
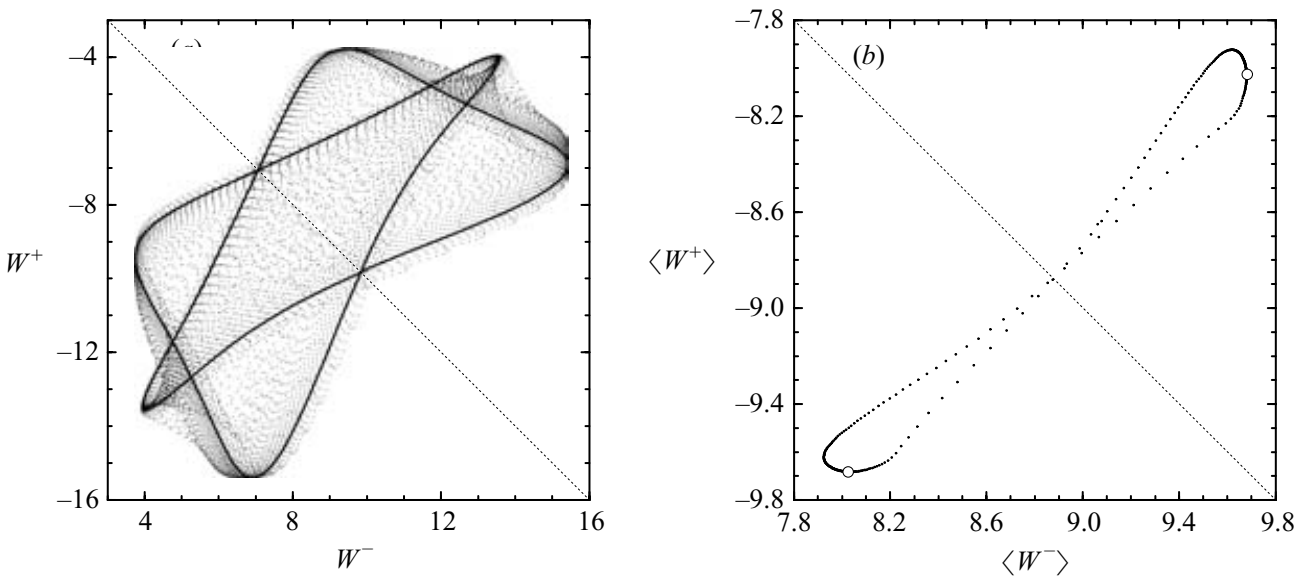

FiguRE 25. Phase portraits of the SNIP transition from $\mathrm{MRW}_{\mathrm{S}}$ to $\mathrm{RW}_{\mathrm{A}}$ at $R e=700$, showing the MRWS for $\Gamma=3.1785$ and the $\mathrm{RW}_{\mathrm{A}}$ for $\Gamma=3.1793$. (a) Full solutions projected on the $\left(W^{-}, W^{+}\right)$plane. $(b)$ Low-pass-filtered solutions projected on the $\left(\left\langle W^{-}\right\rangle,\left\langle W^{+}\right\rangle\right)$.

(central diagram). After the bifurcation the periodic solution no longer exists and there remains a pair of fixed points, one a saddle (unstable, hollow in the figure) and the other a node (stable, solid in the figure). This is a codimension-one bifurcation (Kuznetsov 1998). In the presence of a $Z_{2}$ symmetry, if the limit cycle is not pointwise $Z_{2}$-invariant, then a pair of saddle-nodes appears simultaneously on the invariant circle, as shown schematically in figure $24(b)$. This is what happens in our problem, but instead of a limit cycle we have quasi-periodic solutions (a two-torus), as depicted schematically in figure $24(c)$. In this case the two saddle-nodes become saddle-nodes of limit cycles; the two $Z_{2}$-symmetric saddle-nodes at the bifurcation point are shown as thick lines. Figure 25(a) shows phase portraits of $\mathrm{MRW}_{\mathrm{S}}$ and $\mathrm{RW}_{\mathrm{A}}$ at $R e=700$ to either side of the SNIP bifurcation $\left(\Gamma=3.1785\right.$ for MRWS and 3.1793 for $\left.\mathrm{RW}_{\mathrm{A}}\right)$. In part $(b)$ of this figure are plotted the low-pass-filtered phase portraits of these 

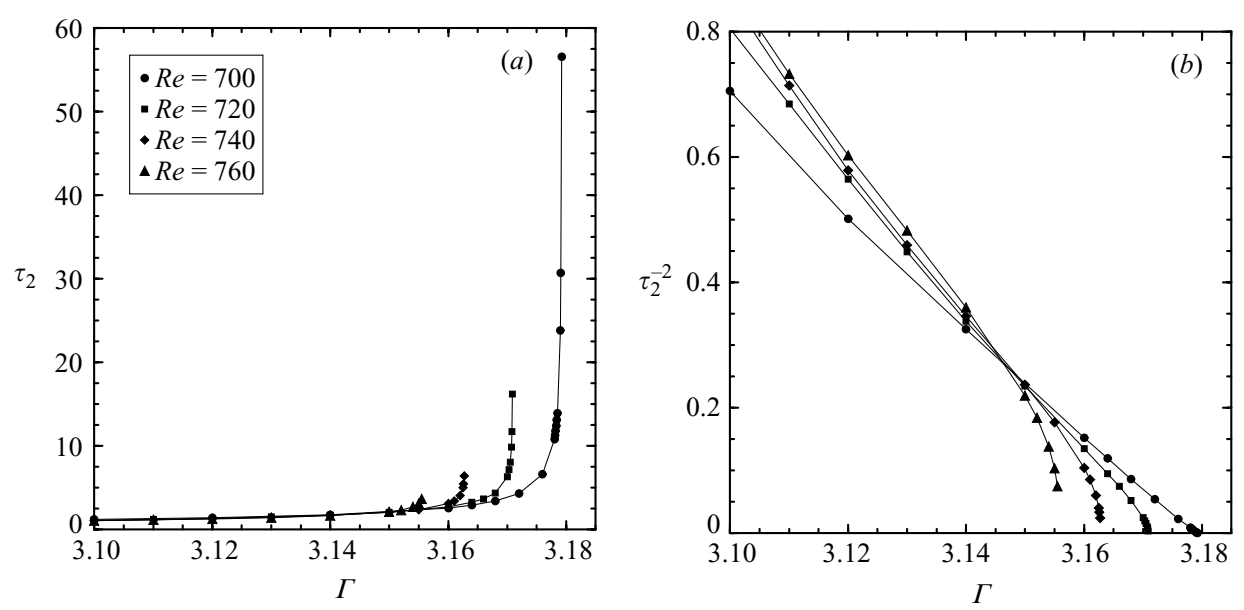

FIGURE 26. Variation with $\Gamma$ of the modulation period, $\tau_{2}$, of MRWs near the SNIP bifurcation, computed for Re as indicated.

solutions showing a situation essentially equivalent to that in the Poincare section of figure 24(c).

As MRWS approaches the SNIP bifurcation, its modulation period $\tau_{2}$ becomes infinite, following the typical inverse square-root law associated with saddle-nodes (Strogatz 1994). This behaviour is illustrated in figure 26. The scaling fits the data best for the lower $R e$ because as $R e$ is increased, there are other bifurcations taking place near the SNIP which affect the modulation period of MRWS (details of these will be presented elsewhere). The primary period, $\tau$, remains close to the precession period of RWS from which MRWS bifurcates, varying by about $10 \%$ over the parameter range considered here.

Focusing on the region where the SNIP, Neimark-Sacker and cyclic pitchfork bifurcation curves approach each other, we find that rather than meeting at a point, there is instead a small window in parameter space where transition between the symmetric and the non-symmetric states is accomplished via a pair of saddle-loop homoclinic (SLH) bifurcations. This window is too small to be seen well in figure 13, so a close-up view is presented in figure 27 . As $\Gamma$ is reduced, $\mathrm{RW}_{\mathrm{A}}$ suffer a NeimarkSacker $\left(\mathrm{NS}_{\mathrm{a}}\right.$ ) bifurcation spawning $Z_{2}$-conjugate $\mathrm{MRW}_{\mathrm{A}}$; the $\mathrm{NS}_{\mathrm{a}}$ bifurcation curve and the SLH bifurcation curve are separated by variations in aspect ratio $\Gamma$ of about $0.03 \%$.

Figures 28(a) and 28(b) show low-pass-filtered phase portraits of the MRW, MRW $_{\mathrm{A}}$, and $\mathrm{RW}_{\mathrm{A}}$ solutions found numerically in such a window. Part $(a)$ shows the projection used earlier: $\left(\left\langle W^{-}\right\rangle,\left\langle W^{+}\right\rangle\right)$. In this projection, the limit cycles are on a two-dimensional manifold that is essentially perpendicular to the projection plane, and so we do not see them very well (this part of the figure consists of MRWS at $\Gamma=3.18062$ and the pair of $Z_{2}$-conjugate $\mathrm{MRW}_{\mathrm{A}}$ at $\Gamma=3.18063$, all at $R e=690$ ). The one good aspect of this projection is that the role of $Z_{2}$ symmetry in these solutions is immediately obvious. A better projection on which to see the phase portraits (although it loses the $Z_{2}$ symmetry information) is $\left(\left\langle U^{+}\right\rangle,\left\langle V^{+}\right\rangle\right)$, where $U^{+}$and $V^{+}$are the radial and azimuthal velocities at the point where the axial velocity $W^{+}$is recorded. Part $(b)$ of the figure is such a projection. The MRWS at $\Gamma=3.18062$ is shown as dots, $\mathrm{MRW}_{\mathrm{A}}$ at $\Gamma$ from 3.18063 to 3.1815 are shown as solid closed curves, and $\mathrm{RW}_{\mathrm{A}}$ at 


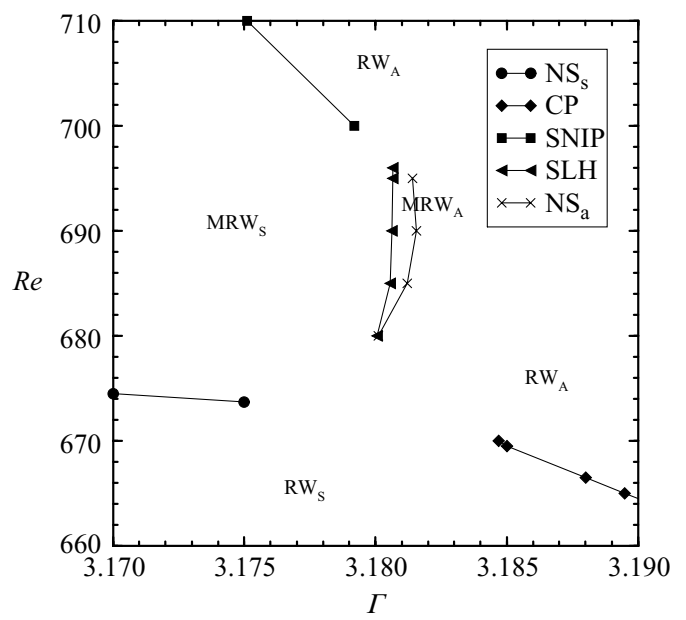

FIGURE 27. Close-up of the numerical bifurcation curves shown in figure 13, focusing in on the SLH bifurcation. Stable solutions in each region are indicated.
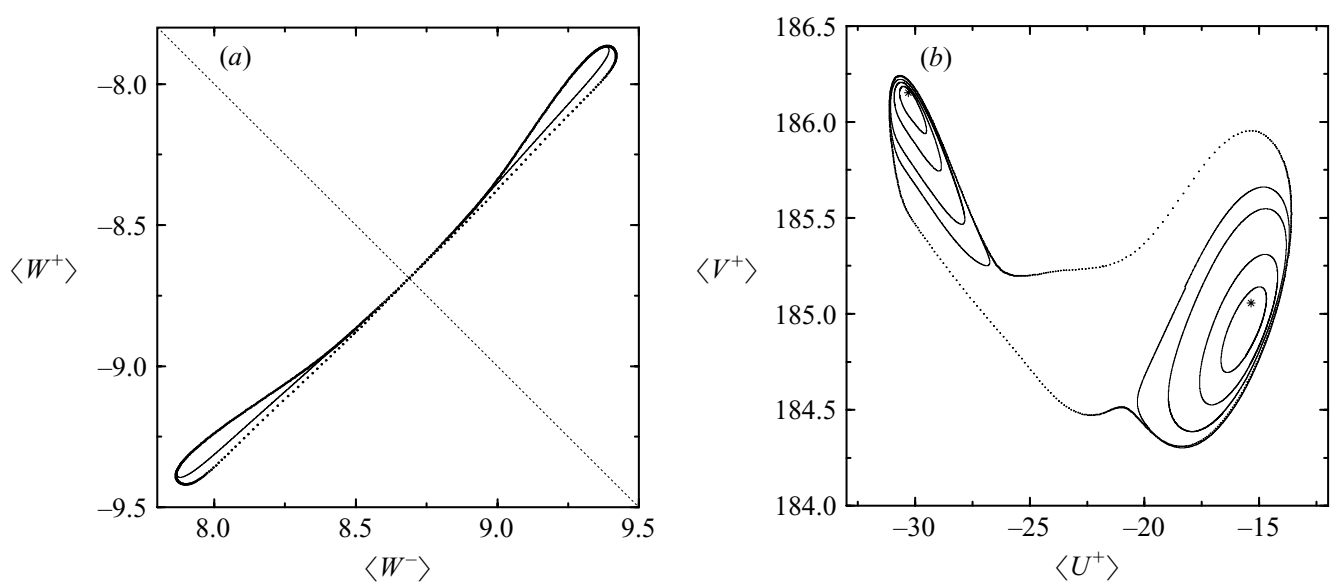

FiguRE 28. (a) Phase portraits projected on $\left(\left\langle W^{-}\right\rangle,\left\langle W^{+}\right\rangle\right)$of MRW at $\Gamma=3.18062$ and MRW $_{\mathrm{A}}$ at $\Gamma=3.18063$, i.e. to either side of the SLH bifurcation. $(b)$ Phase portraits projected on $\left(\left\langle U^{+}\right\rangle,\left\langle V^{+}\right\rangle\right)$of MRWS at $\Gamma=3.18062$, MRW $_{\mathrm{A}}$ at $\Gamma=3.18063,3.1808,3.1812$ and 3.1815 , and $\mathrm{RW}_{\mathrm{A}}$ at 3.1820. All solutions have $R e=690$.

$\Gamma=3.1820$ are the pair of star symbols; all at $R e=690$. In this projection, it is more clear that the pair of $Z_{2}$-conjugate $\mathrm{MRW}_{\mathrm{A}}$ undergoes SLH bifurcations with a pair of $Z_{2}$-conjugate saddle rotating waves (conjectured to appear at a secondary cyclic pitchfork bifurcation from $\mathrm{RW}_{\mathrm{S}}$ ) as $\Gamma$ is reduced from 3.18063. Following the SLH bifurcation, the $Z_{2}$-symmetric MRWS results, which at $\Gamma=3.18062$ is seen to be nearly heteroclinic to the same pair of $Z_{2}$-conjugate saddle rotating waves. The modulation

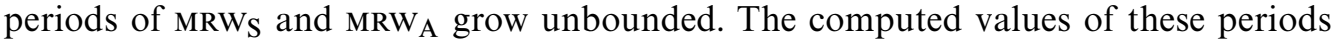
are shown in figure 29, with filled symbols for MRW and open for MRW $\mathrm{M}_{\mathrm{A}}$. One would expect these to following a log scaling law typical of SLH bifurcations (Strogatz 1994). However, the $\log$ fit depends critically on a good estimate of $\Gamma_{\text {crit }}$ (where $\tau_{2} \rightarrow \infty$ ), which is difficult and expensive to obtain numerically. Moreover, the close proximity of the Neimark-Sacker bifurcation $\mathrm{NS}_{\mathrm{a}}$ restricts the range of $\Gamma$ where the scaling 


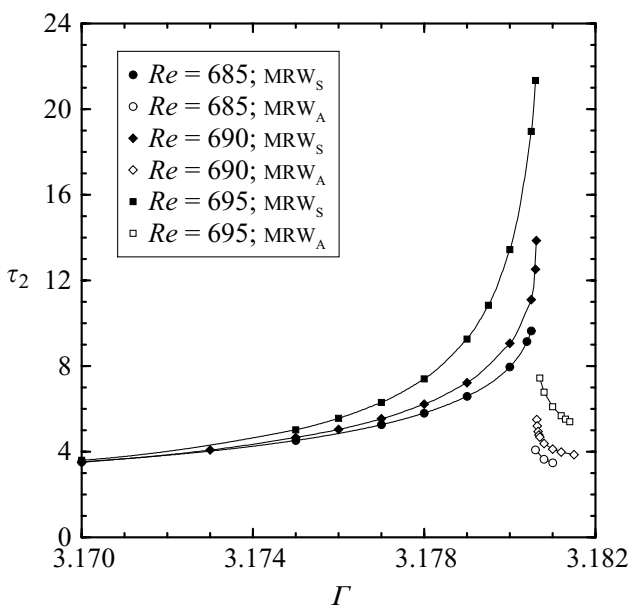

FIGURE 29. Variation with $\Gamma$ of the modulation periods of MRW (filled symbols) and $\mathrm{MRW}_{\mathrm{A}}$ (open symbols) near the SLH bifurcation, computed for $R e$ as indicated.

would hold (one would need to get very close to the SLH bifurcation). For all these reasons, with the data obtained it is not possible to clearly distinguish between a log and an inverse square-root law close to $\Gamma_{\text {crit }}$.

Figure 30 shows the jet dynamics associated with the modulation period $\tau_{2}$ for MRW $_{\mathrm{A}}$ at $R e=690, \Gamma=3.18063$, and $\eta=0.5$. This is the same MRW $\mathrm{A}_{\mathrm{A}}$ as shown in figure 28. The figure shows contours of $v$ in the cylindrical plane $r=r_{i} / d+0.3$, strobed every 38 precession periods; the computed Poincare section is the same as for $\operatorname{MRW}_{\mathrm{S}}\left(U^{+}=-20.0\right)$. The modulation period is $\tau_{2}=5.51$, while the precession period is $\tau=0.0175$; there are $\tau_{2} / \tau=314.8$ precession periods in every modulation period; this ratio is so large because we are very close to the heteroclinic connection. Figure 30 consists of eight frames that are 38 precession periods apart, and hence it nearly covers one modulation period $\tau_{2}$. The main differences with respect to MRWS (see figure 18) are that now the tilt of the upper jet is retarded in $\theta$ with respect to the tilt of the lower jet, while in MRW it was advanced and retarded alternately in time. Moreover, the azimuthal positions of the jets (in the Poincare section) are not fixed. They move in a certain interval of $\theta$. This can be tracked by looking at the positions of the maximum values of $v$ for each jet in the different frames (Poincare sections). The length of the $\theta$-interval for the lower jet is about $56^{\circ}$, whereas for the upper jet it is about $40^{\circ}$, clearly demonstrating the asymmetric nature of $\mathrm{MRW}_{\mathrm{A}}$ with respect to the reflection $z \rightarrow-z$. The $\mathrm{MRW}_{\mathrm{A}}$ exists for a very narrow range in $\Gamma$, and quickly reverts via a Neimark-Sacker bifurcation to a $\mathrm{RW}_{\mathrm{A}}$ with increasing $\Gamma$, in which the oscillation of the relative phase disappears; $\mathrm{RW}_{\mathrm{A}}$ has a fixed phase difference between the tilts of the top and bottom jets.

Details of the bifurcation structure shown in figure 27 are difficult to resolve with the present experimental apparatus. However, experimental evidence is found that the global $Z_{2}$ symmetry-breaking bifurcation from MRWs differs from a SNIP for lower $R e$. Figure 31 show experimental time series of low-pass-filtered LDV measurements of the axial velocity taken at $z=0$ and $r=r_{i}+1.5 \mathrm{~mm}$ for $R e=684.4$ for various $\Gamma$ between 3.162 and 3.171. The data presented have been low-pass filtered with a cut-off frequency of $0.5 \mathrm{~Hz}$ in order to filter out the precession frequency. The time series for $\Gamma=3.162,3.166$ and 3.168 show the very-low-frequency oscillations 

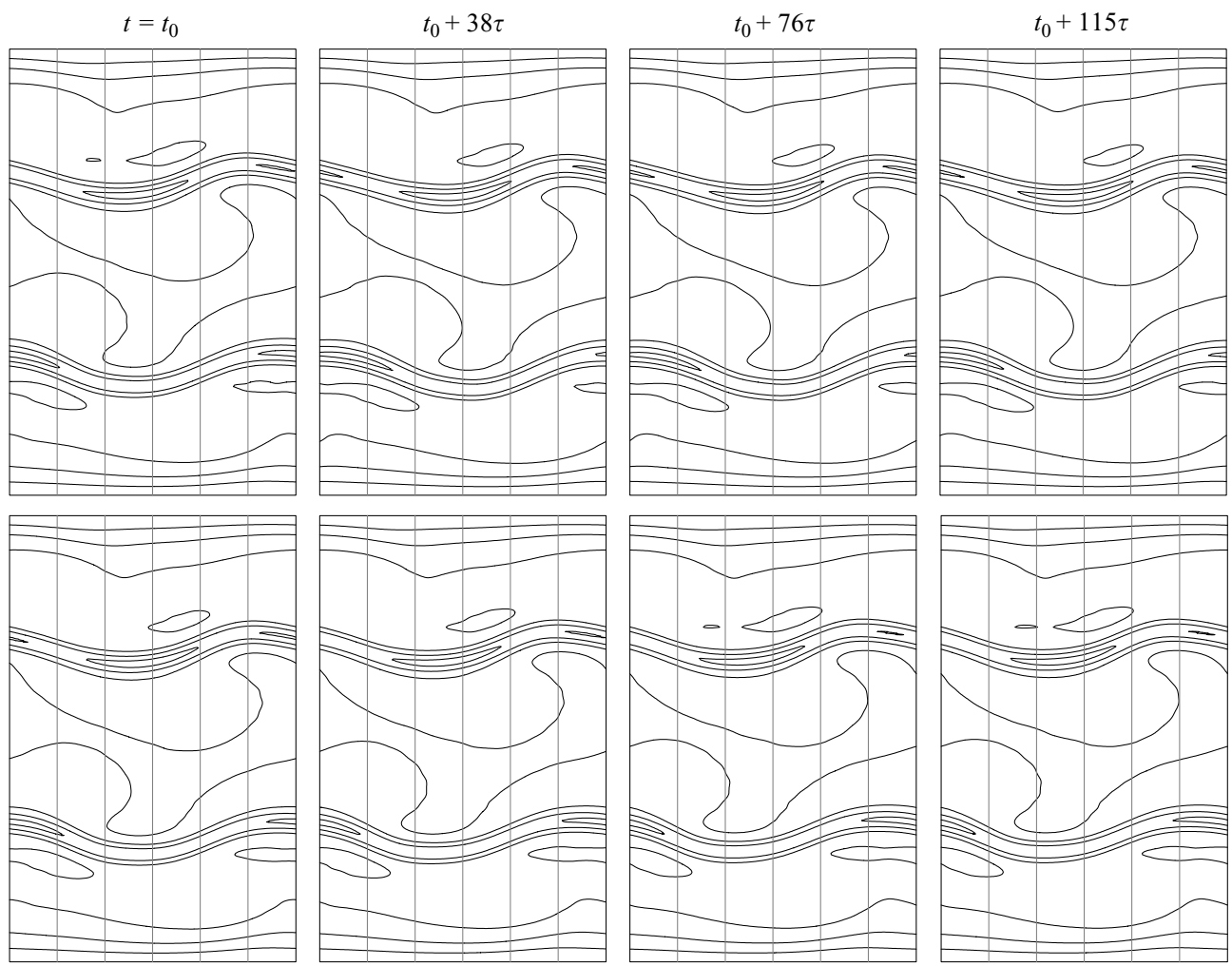

$t_{0}+191 \tau$

$t_{0}+229 \tau$

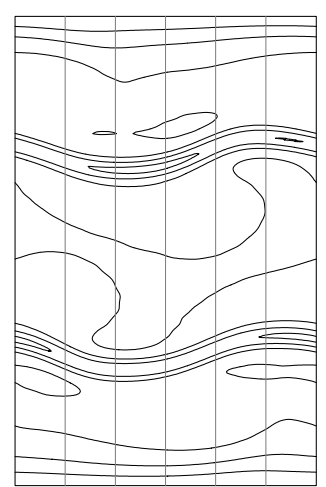

$t_{0}+267 \tau$

FIGURE 30. Contours of $v$ in the cylindrical plane $r=r_{i} / d+0.3$ of $\mathrm{MRW}_{\mathrm{A}}$ at $R e=690$, $\Gamma=3.18063, \eta=0.5$. The sequence covers (approximately) one modulation period $\tau_{2}$, and the frames are 38 precession periods apart $(38 \tau)$. There are twelve equispaced contours with the levels $v \in[0, R e]$. Vertical lines at angles $\theta=j \pi / 3$ for $j=1$ to 5 are included.

$\begin{array}{ccc}\Gamma & \text { Very-low frequency }(\mathrm{Hz}) & \text { Precession frequency }(\mathrm{Hz}) \\ 3.162 & 0.0171 & 4.0039 \\ 3.166 & 0.0134 & 4.0040 \\ 3.168 & 0.0085 & 4.0039 \\ 3.170 & 0.0093 & 4.0035 \\ 3.171 & - & 4.0054\end{array}$

TABLE 2. Frequencies of the flow states in figure 31.

of MRW whose modulation frequency decreases rapidly with decreasing $\Gamma$ as the global bifurcation is approached. The character of the time series at $\Gamma=3.170$ is quite different, but there is still a very-low-frequency oscillation. This state is not space-time $Z_{2}$ symmetric; it is an $\mathrm{MRW}_{\mathrm{A}}$. At $\Gamma=3.171$, the time series is essentially flat (aside from small-amplitude fluctuations due to experimental noise), and the flow state corresponds to $\mathrm{RW}_{\mathrm{A}}$. Table 2 provides the underlying precession periods of all these states as well as the very-low frequencies of the modulated waves. These results provide experimental evidence that the SLH scenario found numerically is physically robust, even though the details are not fully resolved experimentally. 
(a)

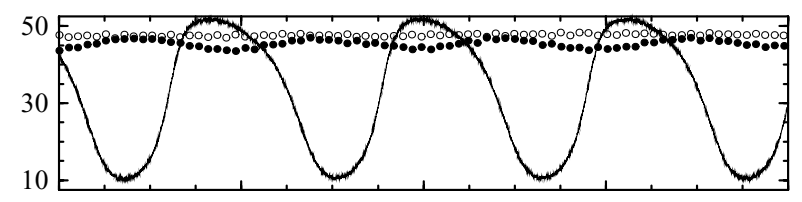

(b)

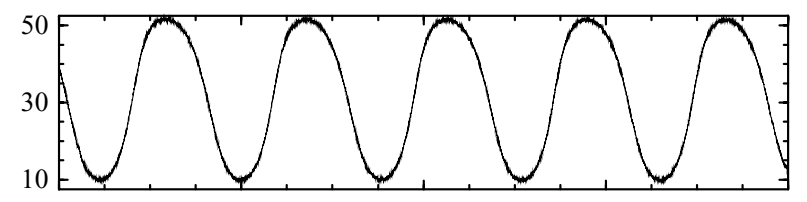

(c)

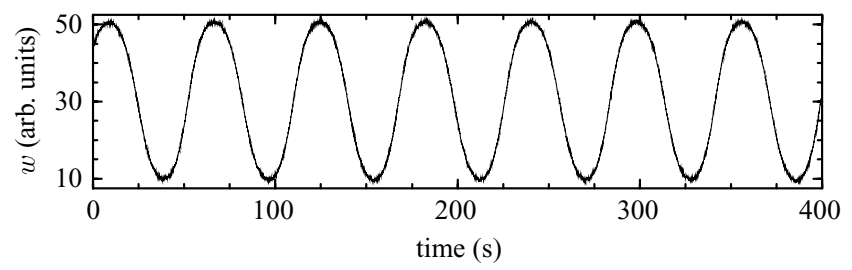

FIGURE 31. Experimentally measured time series (in seconds) of the axial velocity at $R e=684.4$

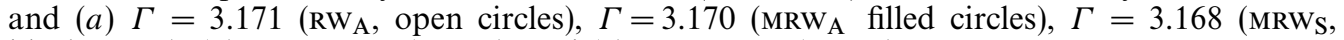
black curve), $(b) \Gamma=3.166\left(\mathrm{MRW}_{\mathrm{S}}\right)$, and $(c) \Gamma=3.162\left(\mathrm{MRW}_{\mathrm{S}}\right)$.

\section{Conclusions}

Unfolding the bifurcation structure of a fluid flow generally provides deep insight into the organizing principles of complex dynamics. Our present study provides a bifurcation study of generic flow states in one of the classical hydrodynamic systems: rotating waves in Taylor-Couette flow. It reveals that rotating waves arising from instabilities of axisymmetric Taylor vortex flow can interact with Taylor vortex flow via a fold-Hopf bifurcation. The fold-Hopf bifurcation is responsible for the appearance of modulated rotating waves whose modulation oscillation develops into a very-low-frequency oscillation as the modulated waves suffer global bifurcations. In the parameter regimes we have studied here, the breaking of the midplane reflection symmetry plays a crucial role in the organization of the global bifurcations and the associated complicated dynamics.

Figure 32 summarizes the experimental and numerical results reported here. Taylor vortex flow solutions with one and two jets, $s_{1}$ and $s_{2}$, exist and are stable inside the broad wedged-shaped region delimited by a pair of saddle-node bifurcation curves which meet at the codimension-two cusp point. $s_{1}$ is stable in the parameter range studied, but $\mathrm{s}_{2}$ loses stability via a symmetry-breaking Hopf bifurcation as $R e$ is increased. The resulting rotating wave $\mathrm{RW}_{\mathrm{S}}$ interacts with the Taylor vortex steady solution $\mathrm{S}_{2}$ at the fold-Hopf bifurcation, and loses stability at a subcritical Neimark-Sacker bifurcation for low $\Gamma$ values. A narrow region of chaotic dynamics exists emerging from the fold-Hopf point, but in the present problem these dynamics are unstable (transient). On increasing $R e$ beyond about 650, the rotating wave $\mathrm{RW}_{\mathrm{S}}$ undergoes two different bifurcations, depending on $\Gamma$. For $\Gamma<3.18$ a Neimark-Sacker bifurcation takes place, resulting in a $Z_{2}$-symmetric two-torus $T_{s}^{2}$ with quasi-periodic solutions MRWs. For $\Gamma>3.18, Z_{2}$ symmetry is broken and a pair of asymmetric limit cycles, $\mathrm{RW}_{\mathrm{A}}$, appear in a cyclic pitchfork bifurcation. 


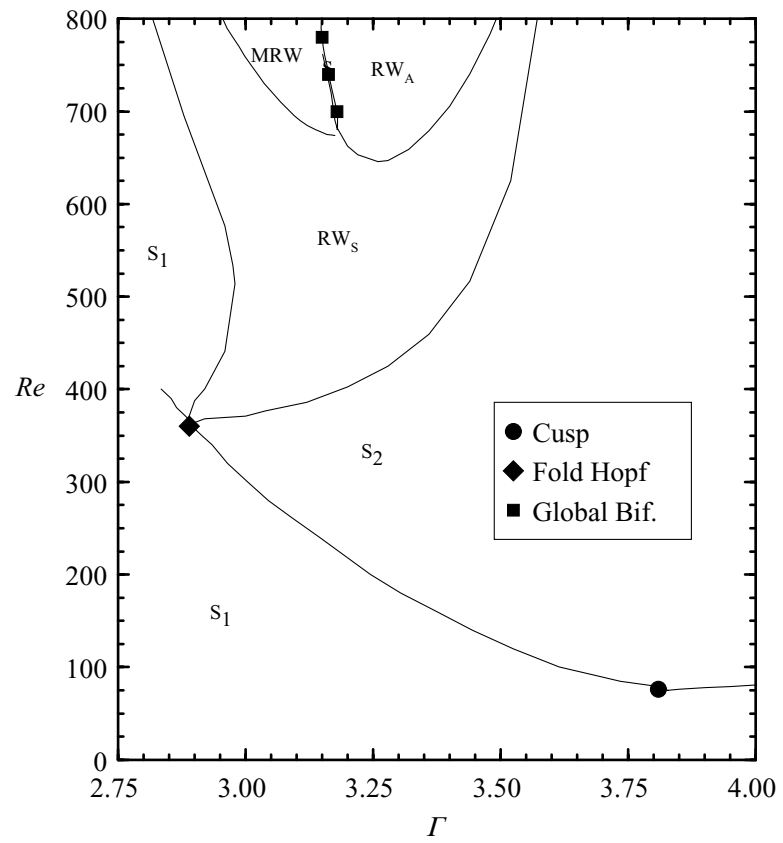

FigURE 32. Numerically determined bifurcation curves shown over a broader parameter range.

Across a curve along $\Gamma \sim 3.18$ a complex symmetry-breaking bifurcation process takes place, providing a connection between the $Z_{2}$-symmetric state (MRWS) and the non- $Z_{2}$-symmetric states (either $\mathrm{RW}_{\mathrm{A}}$ or $\mathrm{MRW}_{\mathrm{A}}$ ). For the connection between $\mathrm{MRW}_{\mathrm{S}}$ and $\mathrm{RW}_{\mathrm{A}}$, this complex process involves a global SNIP (saddle-node-infinite-period) bifurcation with $Z_{2}$ symmetry. Focusing on the region where the SNIP, NeimarkSacker and cyclic pitchfork bifurcation curves approach each other, we find that rather than meeting at a point, there is instead a small window in parameter space where transition between the symmetric and the non-symmetric states is accomplished via a pair of saddle-loop homoclinic (SLH) bifurcations. Computations of the SLH scenario have been presented, and it has also been detected experimentally, providing experimental evidence that the SLH scenario is physically robust, even though the details are not fully resolved experimentally.

It is tempting to speculate that the global bifurcations appearing in the interaction between $\mathrm{RW}_{\mathrm{A}}$ and MRW are in some way associated with the narrow wedge of chaotic dynamics emerging from the fold-Hopf bifurcation; even though these dynamics are unstable at low $R e$, they could manifest themselves when $\mathrm{RW}_{\mathrm{S}}$ becomes unstable at higher $R e$. This conjecture, however, is very difficult to explore. The numerical and experimental tools at our disposal are insufficient for the task.

In general, our results lend further support to the concept of how complex hydrodynamic behaviour results from mode interactions that lead to global bifurcations generating very-low-frequency dynamics. Of course, when present, symmetries are a natural source of multiple modes for the mode interaction, but similar complex behaviour in Taylor-Couette flow in the absence of midplane reflection symmetry has also been observed both experimentally and numerically to be organized by a fold-Hopf bifurcation (e.g. Mullin \& Blohm 2001; Lopez et al. $2004 b$, where the top endwall was stationary and the bottom endwall rotated with the 
inner cylinder). While organization via fold-Hopf bifurcations is quite common over an extensive part of parameter space in Taylor-Couette flows, it is not the exclusive organizing principle. In very-small-aspect-ratio Taylor-Couette flow, for example, Lopez \& Marques (2003) have shown a very-low-frequency mode to be organized via the competition between two rotating waves resulting from a double-Hopf bifurcation.

What is becoming more apparent from the number of studies of bifurcations in Taylor-Couette flows where more than one governing parameter is varied is that there is not a single or even typical route to complex dynamics. Rather, the transition process is as rich and varied as the multiplicity of states that exist (Coles 1965). Nevertheless, the generic codimension-one local bifurcations (the saddle-node and the Hopf) and their codimension-two interactions - the cusp (collision between two saddle-node curves), the fold-Hopf (collision between a saddle-node curve and a Hopf curve), and the double-Hopf (collision between two Hopf curves) - provide the templates organizing much of the observed complex dynamics.

This work was partially supported by grants from Deutsche Forschungsgemeinschaft (G.P. and J. A.), Spanish Ministry of Education and Science (F. M. and J.M. L.), Spanish Ministry of Science and Technology (F. M.), and National Science Foundation (J. M. L.).

\section{REFERENCES}

Abshagen, J., Lopez, J. M., Marques, F. \& Pfister, G. 2005 Symmetry breaking via global bifurcations of modulated rotating waves in hydrodynamics. Phys. Rev. Lett. 94, 074101.

Abshagen, J., Pfister, G. \& Mullin, T. 2001 Gluing bifurcations in a dynamically complicated extended flow. Phys. Rev. Lett. 87, 224501.

Benjamin, T. B. 1978 a Bifurcation phenomena in steady flows of a viscous fluid. Proc. R. Soc. Lond. A 359, 1-26.

Benjamin, T. B. $1978 b$ Bifurcation phenomena in steady flows of a viscous fluid. II. Experiments. Proc. R. Soc. Lond. A 359, 27-43.

Benjamin, T. B. \& Mullin, T. 1982 Notes on the multiplicity of flows in the Taylor experiment. J. Fluid Mech. 121, 219-230.

Blackburn, H. M. \& Lopez, J. M. 2002 Modulated rotating waves in an enclosed swirling flow. J. Fluid Mech. 465, 33-58.

Chossat, P. \& Iooss, G. 1994 The Couette-Taylor Problem. Springer.

Coles, D. 1965 Transition in circular Couette flow. J. Fluid Mech. 21, 385-425.

Crawford, J. D. \& KNobloch, E. 1991 Symmetry and symmetry-breaking bifurcations in fluid dynamics. Annu. Rev. Fluid Mech. 23, 341-387.

DiPrima, R. C. \& Swinney, H. L. 1981 Instabilities and transition in flow between concentric rotating cylinders. In Hydrodynamics Instabilities and the Transtion to Turbulence (ed. H. L. Swinney \& J. P. Gollub). Topics in Applied Physics, vol. 45. Springer.

Egbers, C. \& Pfister, G. (Eds.) 2000 Physics of Rotating Fluids. Lecture Notes in Physics, vol. 549. Springer.

Gerdts, U., von Stamm, J., Buzug, T. \& Pfister, G. 1994 Axisymmetric time-dependent flow in the Taylor-Couette system. Phys. Rev. E 49, 4019-4026.

Guckenheimer, J. \& Holmes, P. 1983 Nonlinear Oscillations, Dynamical Systems, and Bifurcations of Vector Fields. Springer.

Iooss, G. \& Adelmeyer, M. 1998 Topics in Bifurcation Theory and Applications, 2nd edn. World Scientific.

Kevrekidis, I. G., Rico-Martínez, R., Ecke, R. E., Farber, R. M. \& Lapedes, A. S. 1994 Global bifurcations in Raleigh-Bénard convection. Experiments, empirical maps and numerical bifurcation analysis. Physica D 71, 342-362.

KNobloch, E. 1994 Bifurcations in rotating systems. In Lectures on Solar and Planetary Dynamos (ed. M. R. E. Proctor \& A. D. Gilbert), pp. 331-372. Cambridge University Press. 
Krupa, M. 1990 Bifurcations of relative equilibria. SIAM J. Math. Anal. 21, 1453-1486.

Kuznetsov, Y. A. 1998 Elements of Applied Bifurcation Theory, 2nd edn. Springer.

Lopez, J. M., Hart, J. E., Marques, F., Kittelman, S. \& Shen, J. $2002 a$ Instability and mode interactions in a differentially-driven rotating cylinder. J. Fluid Mech. 462, 383-409.

Lopez, J. M. \& Marques, F. 2003 Small aspect ratio Taylor-Couette flow: Onset of a very-lowfrequency three-torus state. Phys. Rev. E 68, 036302.

Lopez, J. M. \& Marques, F. 2004 Mode competition between rotating waves in a swirling flow with reflection symmetry. J. Fluid Mech. 507, 265-288.

Lopez, J. M., Marques, F. \& Fernando, H. J. S. (Eds.) 2004a Theoret. Comput. Fluid Dyn.; special issue Taylor-Couette Flows, vol. 18(2-4). Springer.

Lopez, J. M., Marques, F. \& Shen, J. $2002 b$ An efficient spectral-projection method for the NavierStokes equations in cylindrical geometries II. Three dimensional cases. J. Comput. Phys. 176, 384-401.

Lopez, J. M., Marques, F. \& Shen, J. 2004b Complex dynamics in a short Taylor-Couette annulus with the top endwall stationary and the bottom rotating. J. Fluid Mech. 501, 327-354.

LOPEZ, J. M. \& SHEN, J. 1998 An efficient spectral-projection method for the Navier-Stokes equations in cylindrical geometries I. Axisymmetric cases. J. Comput. Phys. 139, 308-326.

Marques, F., Lopez, J. M. \& Shen, J. 2002 Mode interactions in an enclosed swirling flow: a double Hopf bifurcation between azimuthal wavenumbers 0 and 2. J. Fluid Mech. 455, 263-281.

MulLin, T. 1982 Mutations of steady cellular flows in the Taylor experiments. J. Fluid Mech. 121, 207-218.

Mullin, T. 1991 Finite-dimensional dynamics in Taylor-Couette flow. IMA J. Appl. Maths 46, $109-119$.

Mullin, T. 1993 The Nature of Chaos. Oxford University Press.

Mullin, T. \& Blohm, C. 2001 Bifurcation phenomena in a Taylor-Couette flow with asymmetric boundary conditions. Phys. Fluids 13, 136-140.

Mullin, T., Tavener, S. J. \& Cliffe, K. A. 1989 An experimental and numerical study of a codimension-2 bifurcation in a rotating annulus. Europhys. Lett. 8, 251-256.

Mullin, T., Toya, Y. \& TAVEner, S. J. 2002 Symmetry breaking and multiplicity of states in small aspect ratio Taylor-Couette flow. Phys. Fluids 14, 2778-2787.

Nore, C., Tuckerman, L. S., Daube, O. \& Xin, S. 2003 The 1:2 mode interaction in exactly counter-rotating von Karman swirling. J. Fluid Mech. 477, 51-88.

Peacock, T. \& Mullin, T. 2001 Homoclinic bifurcations in a liquid crystal flow. J. Fluid Mech. 432, 369-386.

Price, T. J. \& Mullin, T. 1991 An experimental observation of a new type of intermittency. Physica D 48, 29-52.

von Stamm, J., Gerdts, U., Buzug, T. \& Pfister, G. 1996 Symmetry breaking and period doubling on a torus in the VLF regime in Taylor-Couette flow. Phys. Rev. E 54, 4938-4957.

Strogatz, S. 1994 Nonlinear Dynamics and Chaos. Addison-Wesley.

Swift, J. W. \& Wiesenfeld, K. 1984 Suppression of period doubling in symmetric systems. Phys. Rev. Lett. 52, 705-708.

TAGG, R. P. 1994 The Couette-Taylor problem. Nonlinear Sci. Today 4, 1-25.

Tuckerman, L. S. \& BARKLeY, D. 1988 Global bifurcation to traveling waves in axisymmetric convection. Phys. Rev. Lett. 61, 408-411.

WigGins, S. 1988 Global Bifurcations and Chaos. Springer. 